TRANSACTIONS OF THE

AMERICAN MATHEMATICAL SOCIETY

Volume 357, Number 10, Pages 3865-3886

S 0002-9947(05)03968-1

Article electronically published on May 20, 2005

\title{
RAMSEY FAMILIES OF SUBTREES OF THE DYADIC TREE
}

\author{
VASSILIS KANELLOPOULOS
}

\begin{abstract}
We show that for every rooted, finitely branching, pruned tree $T$ of height $\omega$ there exists a family $\mathcal{F}$ which consists of order isomorphic to $T$ subtrees of the dyadic tree $C=\{0,1\}^{<\mathbb{N}}$ with the following properties: (i) the family $\mathcal{F}$ is a $G_{\delta}$ subset of $2^{C}$; (ii) every perfect subtree of $C$ contains a member of $\mathcal{F}$; (iii) if $K$ is an analytic subset of $\mathcal{F}$, then for every perfect subtree $S$ of $C$ there exists a perfect subtree $S^{\prime}$ of $S$ such that the set $\left\{A \in \mathcal{F}: A \subseteq S^{\prime}\right\}$ either is contained in or is disjoint from $K$.
\end{abstract}

\section{INTRODUCTION}

Ramsey theory for trees originated from the fundamental Halpern-Läuchli Theorem [HL] and its versions due independently to R. Laver and D. Pincus (see also AFK], [H] $[\mathrm{HP}],[\mathrm{L}], \mathrm{M} 2],[\mathrm{C}]$ and $[\mathrm{CS}]$ ). Among the first Ramsey results for trees were the partition theorems for strong subtrees due to K. Milliken [M1], M2]. His methods relied upon the strong subtree version of Halpern-Läuchli Theorem as well as the work of Galvin-Prikry [GP and E. Ellentuck [E]. Strongly related to trees are the partition theorems for subsets of perfect sets in the real line. F. Galvin $\mathrm{G}$ ] initiated this research by proving a Ramsey result concerning two-element subsets of perfect sets and making a conjecture for Borel partitions of $n$-element subsets, for $n \geq 3$. This conjecture was settled by A. Blass $[\mathrm{B}$. A. Louveau, S. Shelah and B. Veličković [LSV] later extended Blass' results for infinite subsets of perfect sets. Their result concerns analytic partitions of infinite downwards closed subtrees of the dyadic tree of a certain type. As a corollary they gave alternative proofs of parameterized versions of Galvin-Blass' theorem as well as of Stern's theorem [St] for analytic partitions of chains of the dyadic tree. An excellent presentation of the above results as well as consequences of them is contained in [T2]. Moreover, applications of the parameterized Ramsey theory can be found in [T1].

A natural question can be posed here. Is it possible to have a Ramsey result for partitions of subtrees of the dyadic tree which are not necessarily downwards closed? That is, a result like Stern's but for more complicated subtrees? Milliken's theorem is a result of this form concerning strong subtrees, which is a rather restricted class of subtrees.

The aim of the present paper is to provide an answer to the above question. More precisely, we prove a Ramsey result for subtrees of the dyadic tree which are not downwards closed and moreover they are order isomorphic to an arbitrary rooted, pruned and finitely branching tree of height $\omega$. Our result simultaneously extends

Received by the editors August 5, 2002.

2000 Mathematics Subject Classification. Primary 05C05.

This research was partially supported by the Thales program of NTUA.

(c)2005 American Mathematical Society Reverts to public domain 28 years from publication 
Louveau-Shelah-Veličković's theorem as well as Stern's theorem for broader classes of subtrees of the dyadic tree.

Before stating our result, let us fix some terminology. By the term tree we mean a tree of height $\omega$, which is rooted, has no maximal elements and is finitely branching. Two trees $S$ and $T$ are called order isomorphic if there exists an order preserving bijection $\phi: S \rightarrow T$. By $C$ we denote the dyadic tree of finite sequences of 0 's and 1's ordered by the initial segment ordering denoted by $<$. A subtree $T$ of $C$ is called perfect if every $t \in T$ has at least two incomparable successors in $T$. A family $\mathcal{F}$ of subtrees of $C$ is said to be perfectly dense if for every perfect subtree $T$ of $C$, there exists an $A \in \mathcal{F}$ such that $A \subseteq T$. A subset $X$ of $\mathcal{F}$ is called perfectly Ramsey if for every perfect subtree $T$ of $C$ there exists a perfect subtree $T^{\prime} \subseteq T$ such that either $\left\{A \in \mathcal{F}: A \subseteq T^{\prime}\right\} \subseteq X$ or $\left\{A \in \mathcal{F}: A \subseteq T^{\prime}\right\} \cap X=\emptyset$.

A family $\mathcal{F}$ of subtrees of $C$ is called a Ramsey family if the following are satisfied:

(1) $\mathcal{F}$ is a $G_{\delta}$ subset of $2^{C}$.

(2) $\mathcal{F}$ is perfectly dense.

(3) Every analytic subset of $\mathcal{F}$ is perfectly Ramsey.

Using the above definitions, our main theorem is stated as follows:

Theorem A. For every tree $T$ there exists a Ramsey family $\mathcal{F}$ consisting of order isomorphic to $T$ subtrees of $C$.

Roughly speaking, the Ramsey family $\mathcal{F}$ satisfying the conclusion of Theorem A is of the following form. First it consists of subtrees of $C$ which are isomorphic to each other in a stronger sense. Namely, for every $S, S^{\prime} \in \mathcal{F}$ there exists a bijection $\phi: S \rightarrow S^{\prime}$ preserving both orders $<$ and $\prec$, where by $\prec$ we denote the lexicographic ordering of $C$. This is a necessary restriction in order to avoid certain pathologies due to the lexicographic ordering of $C$. To classify such isomorphic classes we introduce the notion of the coding $f$ of a subtree $S$ of $C$ (see Definitions 12). This is a function defined on $\mathbb{N}$ and taking values in $\mathbb{N}^{\mathbb{N}}$, such that for every $n, f(n)$ shows the cardinality of the set of immediate successors of every node contained in the $n^{\text {th }}$ level $S(n)$ of $S$. We could say that the coding is the analogue of the notion of the type introduced in [LSV]. The difference is that the type refers primarily to downwards closed skew subtrees of $C$ and also to subtrees of the same type that are not necessarily order isomorphic to each other. The type rather characterizes the set of branches of the subtree (the body of the subtree) and not the structure of the subtree itself.

Returning to the description of $\mathcal{F}$, its second property is that it consists of subtrees of $C$ of a special family, which we call the family of rapidly increasing subtrees of $C$ (see Definition 14). As we will show, the family of rapidly increasing subtrees of a given coding $f$ is perfectly dense. In the trivial case of a coding of a subtree without splitting nodes, this family coincides with the family of all chains of $C$. For every rapidly increasing subtree $S$, we define its regular envelope $\bar{S}$, which is the minimal regular subtree of $C$ containing $S$. (A subtree $R$ of a tree $T$ is called regular if for every $n$ there exists $m$ such that $R(n) \subset T(m)$.) It turns out that the regular envelope of a rapidly increasing subtree of $C$ is a skew subtree of $C$, that is, a subtree with at most one splitting node in every level and such that every splitting node of it has exactly two immediate successors. Moreover, if the coding $f$ of a rapidly increasing subtree $S$ is given, then there exists a unique skew tree coding $\bar{f}$ such that the regular envelope $\bar{S}$ is of coding $\bar{f}$. In particular, we show 
that the family of rapidly increasing subtrees of coding $f$ is homeomorphic to the family of the regular skew subtrees of coding $\bar{f}$. This property of rapidly increasing subtrees is one of the keys of the proof of our result since the regular skew trees are easier to deal with.

The proof of Theorem A follows the lines of abstract Ramsey theory due to S. Todorčević T2. We refer to the basics of this theory briefly in the paper. This theory deals with two spaces $\mathcal{R}$ and $\mathcal{S}$ with approximations and which satisfy certain axioms. The main theorem is Theorem 33. One of the main differences from the well-known theory of T.J. Carlson [C] is that the basic sets do not necessarily form a basis for a topology. This causes several difficulties for the proof that Ramsey subsets are closed under the Souslin operation.

As it is standard in infinite Ramsey theory, at a certain point of the proof we need an extension argument, which is actually a finite Ramsey-type result. This is clarified in the form of an axiom in the abstract Ramsey theory. In Ramsey theory for infinite trees, this finite-type result is always related to the Halpern-Läuchli Theorem and its variants. The same holds in our approach, and the key ingredient of our proofs is a consequence of the Halpern-Läuchli Theorem.

To state it we need some definitions. For a finite sequence $T, S_{1}, \ldots, S_{d}$ of dyadic trees, we define the "two-one" level product as follows. For each $n$, we set $T^{(2)}(n)$ to be the set of all doubletons of $T(n)$, i.e.

$$
T^{(2)}(n)=\left\{\left\{t_{1}, t_{2}\right\}: t_{1}, t_{2} \in T(n) \text { and } t_{1} \neq t_{2}\right\} .
$$

The "two-one" level product of $T, S_{1}, \ldots, S_{d}$ is defined to be the set

$$
\otimes\left(T^{(2)}, S_{1}, \ldots, S_{d}\right)=\bigcup_{n} T^{(2)}(n) \times S_{1}(n) \times \cdots \times S_{d}(n) .
$$

Also, we use the following notation. If $S$ is a regular subtree of $T$, then we define the level set of $S$ in $T$ to be the set $\left\{m_{n}: S(n) \subseteq T\left(m_{n}\right), n \in \mathbb{N}\right\}$. We prove the following partition theorem.

Theorem B. Let $T, S_{1}, \ldots, S_{d}$ be a finite sequence of dyadic trees and suppose that $\otimes\left(T^{(2)}, S_{1}, \ldots, S_{d}\right)$ is finitely colored. Then there exist regular dyadic subtrees $T^{\prime}, S_{1}^{\prime}, \ldots, S_{d}^{\prime}$ of $T, S_{1}, \ldots, S_{d}$ with the same level set in $T, S_{1}, \ldots, S_{d}$ respectively, such that $\otimes\left(T^{\prime(2)}, S_{1}^{\prime}, \ldots, S_{d}^{\prime}\right)$ is monochromatic.

The perfect tree version of the above theorem will be used to verify the crucial final axiom of the abstract Ramsey theory.

The paper is organized as follows. In section 2 , we prove Theorem B. In section 3 , we give the definitions of the coding and of the family of rapidly increasing subtrees of the dyadic tree $C$. In section 4 , we state Todorčević's abstract Ramsey theory, and in the final section we prove our main results.

\section{The "TWO-ONE" LEVEL PRODUCT OF A FINITE SEQUENCE OF PERFECT TREES}

2.1. Definitions and notations. By $\mathbb{N}$ we denote the set of non-negative integers. For a set $X$, by $|X|$ we denote the cardinality of the set.

Let $(T, \leq)$ be a partially ordered set. For each $t \in T$ we define the set of predecessors of $t$ in $T$ to be the set Pred $(t, T)=\left\{t^{\prime}: t^{\prime}<t\right\}$ and the set of successors of $t$ in $T$ to be the set $\operatorname{Succ}(t, T)=\left\{t^{\prime}: t<t^{\prime}\right\}$. Generally, a tree $T$ is defined to 
be a partially ordered set $(T, \leq)$, such that for all $t \in T$ the set Pred $(t, T)$ is well ordered by $\leq$. The elements of a tree are called nodes.

We are interested in trees $T$, such that Pred $(t, T)$ is finite for all $t \in T$. For such trees $T$ and $t \in T$ let $|t|_{T}=|\operatorname{Pred}(t, T)|$ be the order of $t$ in $T$ and for each $n \in \omega$, let $T(n)=\left\{t \in T:|t|_{T}=n\right\}$ be the $n^{\text {th }}$-level of $T$. For each $t \in T$, we define the set of immediate successors of $t$ in $T$ to be the set $\operatorname{ImmSucc}(t, T)=$ $\left\{t^{\prime} \in T: t^{\prime}>t\right.$ and $\left.\left|t^{\prime}\right|_{T}=|t|_{T}+1\right\}$. A tree $T$ will be called finitely branching if for every $t \in T$ the set $\operatorname{ImmSucc}(t, T)$ is finite. A branch of a tree $T$ is a maximal chain in $(T, \leq)$.

The minimal nodes of a tree are called roots of the tree, and if the tree $T$ has a unique root, then we will call $T$ a rooted tree. The ordinal $h(T)=\sup \left\{|t|_{T}+1: t \in\right.$ $T\}$ is called the height of $T$. We are only concerned with trees of height at most $\omega$. For such trees $T$ and for every $L \subset \mathbb{N}$ we set $\left.T\right|_{L}=\bigcup_{n \in L} T(n)$. A tree is called pruned if every node of it has at least one immediate successor.

A node of a tree is called splitting if it has at least two immediate successors. A tree $T$ is called dyadic if it has a unique root, $h(T)=\omega$, and every node of it has exactly two immediate successors. A tree $T$ is called perfect if for every $t \in T$ there is a $t^{\prime} \in T$, with $t \leq t^{\prime}$, such that $t^{\prime}$ is a splitting node. A pruned tree $T$ is called skew if every splitting node of $T$ has exactly two immediate successors and every level of $T$ contains at most one splitting node.

If $T$ is a tree, then every subset $S$ of $T$ is called a subtree of $T$. If $S$ is a subtree of $T$, then the set $\{m \in \mathbb{N}: T(m) \cap S \neq \varnothing\}$ is called the level set of $S$ in $T$ and will be denoted by $L_{T}(S)$. A subtree $S$ of a tree $T$ is called a regular subtree of $T$ if for each $n \in h(S)$ there is an $m_{n} \in h(T)$ such that $S(n) \subseteq T\left(m_{n}\right)$.

We will write $S \preceq T$ to denote that $S$ is a regular subtree of $T$. Clearly if $S^{\prime} \preceq S \preceq T$, then $S^{\prime} \preceq T$. Also if $S^{\prime}, S$ are regular subtrees of $T$ and $S^{\prime}$ is a subtree of $S$, then $S^{\prime} \preceq S$.

A subtree $S$ of $T$ is called a strong subtree of $T$ if it is a regular subtree of $T$ and in addition satisfies the following condition: for every $s \in S$ and $t \in \operatorname{ImmSucc}(s, T)$ the set $T_{t} \cap \operatorname{ImmSucc}(s, S)$ is a singleton, where $T_{t}=\left\{t^{\prime} \in T: t^{\prime} \geq t\right\}$.

For $t_{1}, t_{2} \in T$, we set $t_{1} \wedge_{T} t_{2}$ to be the infimum of the set $\left\{t_{1}, t_{2}\right\}$ in $T$. Observe that if $S$ is a strong subtree of $T$, then for every $t_{1}, t_{2} \in S, t_{1} \wedge_{T} t_{2}=t_{1} \wedge_{S} t_{2}$.

A $d$-tuple of trees $\left(T_{1}, \ldots, T_{d}\right)$, such that $T_{1}, \ldots, T_{d}$ are of common height $h \leq \omega$, will be called a $d$-vector tree of height $h$ or simply a vector tree. A vector tree $\left(S_{1}, \ldots, S_{d}\right)$ will be called a vector subtree of $\left(T_{1}, \ldots, T_{d}\right)$ if for each $i=1, \ldots, d, S_{i}$ is a subtree of $T_{i}$.

A vector subtree $\left(S_{1}, \ldots, S_{d}\right)$ will be called a regular vector subtree of $\left(T_{1}, \ldots, T_{d}\right)$ if $S_{i}$ is a regular subtree of $T_{i}$ and there is an $L \subset \mathbb{N}$ such that $L=L_{T_{i}}\left(S_{i}\right)$, for all $i=1, \ldots, d$. In this case, the set $L$ will be called the level set of $\left(S_{1}, \ldots, S_{d}\right)$ in $\left(T_{1}, \ldots, T_{d}\right)$. In particular, a regular vector subtree $\left(S_{1}, \ldots, S_{d}\right)$ of $\left(T_{1}, \ldots, T_{d}\right)$ is called a strong vector subtree of $\left(T_{1}, \ldots, T_{d}\right)$ if for all $i=1, \ldots, d, S_{i}$ is a strong subtree of $T_{i}$. The regular vector subtrees $\left(S_{1}, \ldots, S_{d}\right)$ and $\left(S_{1}^{\prime}, \ldots, S_{d}^{\prime}\right)$ of $\left(T_{1}, \ldots, T_{d}\right)$ are called compatible in $\left(T_{1}, \ldots, T_{d}\right)$ if they have the same level set in $\left(T_{1}, \ldots, T_{d}\right)$.

Vector trees will be denoted by capital bold letters, such as $\mathbf{T}, \mathbf{S}, \mathbf{R}, \ldots$ If $\mathbf{S}=\left(S_{1}, \ldots, S_{d}\right)$ is a regular vector subtree of $\mathbf{T}=\left(T_{1}, \ldots, T_{d}\right)$, then we will denote it by $\mathbf{S} \preceq \mathbf{T}$. Also, when we say that $\mathbf{T}=\left(T_{1}, \ldots, T_{d}\right)$ is a perfect (resp. dyadic) vector tree, then we will mean that for every $i=1, \ldots, d, T_{i}$ is a perfect (resp. dyadic) tree. 
If $\mathbf{T}=\left(T_{1}, \ldots, T_{d}\right)$ is a vector tree of height $\omega$, then the level product of $\mathbf{T}=$ $\left(T_{1}, \ldots, T_{d}\right)$ is defined to be the set

$$
\otimes \mathbf{T}=\otimes\left(T_{1}, \ldots, T_{d}\right)=\bigcup_{n} T_{1}(n) \times \cdots \times T_{d}(n) .
$$

We will need the next consequence of the Halpern-Läuchli Theorem, which was also proved independently by Laver and Pincus (unpublished). For a proof the reader can consult [AFK].

Theorem 1. Let $\left(T_{1}, \ldots, T_{d}\right)$ be a vector tree and suppose that $\otimes\left(T_{1}, \ldots, T_{d}\right)$ is finitely colored. Then there exists a strong vector subtree $\left(S_{1}, \ldots, S_{d}\right)$ of $\left(T_{1}, \ldots, T_{d}\right)$ such that $\otimes\left(S_{1}, \ldots, S_{d}\right)$ is monochromatic.

Observe that if $\left(T_{1}, \ldots, T_{d}\right)$ is a dyadic vector tree, then every strong vector subtree of it is also a dyadic vector subtree. The above theorem will be referred to in the sequel as the HLLP Theorem.

2.2. The main theorem. Let $\left(T, S_{1}, \ldots, S_{d}\right)$ be a dyadic vector tree. For each $n$, let $T^{(2)}(n)$ be the set of all unordered pairs of $T(n)$, i.e.

$$
T^{(2)}(n)=\left\{\left\{t_{1}, t_{2}\right\}: t_{1} \neq t_{2} \text { and } t_{1}, t_{2} \in T(n)\right\} .
$$

(For $n=0, T^{(2)}(0)=\varnothing$.) Then the two-one level product of $\left(T, S_{1}, \ldots, S_{d}\right)$ is defined as follows:

$$
\otimes\left(T^{(2)}, S_{1}, \ldots, S_{d}\right)=\bigcup_{n} T^{(2)}(n) \times S_{1}(n) \times \cdots \times S_{d}(n) .
$$

Our goal in this section is to prove the following partition theorem.

Theorem 2. Let $\left(T, S_{1}, \ldots, S_{d}\right)$ be a dyadic vector tree and let $\otimes\left(T^{(2)}, S_{1}, \ldots, S_{d}\right)$ be finitely colored. Then there exists a regular dyadic vector subtree $\left(T^{\prime}, S_{1}^{\prime}, \ldots, S_{d}^{\prime}\right)$ of $\left(T, S_{1}, \ldots, S_{d}\right)$ such that $T^{\prime}$ is a strong subtree of $T$ and $\otimes\left(T^{\prime(2)}, S_{1}^{\prime}, \ldots, S_{d}^{\prime}\right)$ is monochromatic.

Before passing to the proof let us make some comments concerning possible extensions of the above theorem. Perhaps one can think that corresponding results could hold for a "three-one" or more generally a " $n$-one" level product. But this fails because of the appearance of different types of $n$-tuples $\left(t_{1}, \ldots, t_{n}\right)$ for $n \geq 3$. Galvin first noticed this in his conjecture related to partitions of finite subsets of perfect sets, a conjecture that latter was settled by Blass $[\mathrm{B}]$.

Similar difficulties arise for, let us say, "two-two" level products. For example, let $T, S$ be dyadic trees and define

$$
\otimes\left(T^{(2)}, S^{(2)}\right)=\bigcup_{n} T^{(2)}(n) \times S^{(2)}(n) .
$$

Let us color the tuple $\left(t_{1}, t_{2}, s_{1}, s_{2}\right)$ in the above product "red" if

$$
\left|t_{1} \wedge_{T} t_{2}\right|_{T}>\left|s_{1} \wedge_{S} s_{2}\right|_{S}
$$

and color it "blue" otherwise. It is not difficult to verify that for any regular dyadic vector subtree $\left(T^{\prime}, S^{\prime}\right)$ of $(T, S)$, the level product $\otimes\left(T^{\prime(2)}, S^{\prime(2)}\right)$ contains both colors. 
Proof of Theorem 2. In the sequel we will use some notational conventions. If $\mathbf{S}=$ $\left(S_{1}, \ldots, S_{d}\right)$ is a $d$-vector tree and $T$ is a tree, then we write $(T, \mathbf{S})$ instead of $\left(T, S_{1}, \ldots, S_{d}\right)$. Also the level product of $\left(T, S_{1}, \ldots, S_{d}\right)$ will be denoted by $\otimes(T, \mathbf{S})$ and the "two-one" level product by $\otimes\left(T^{(2)}, \mathbf{S}\right)$. The elements of $\otimes\left(T^{(2)}, \mathbf{S}\right)$ will be denoted by small bold letters such as $\mathbf{t}$. For a vector subtree $\mathbf{S}^{\prime}=\left(S_{1}^{\prime}, \ldots, S_{d}^{\prime}\right)$ of $\mathbf{S}$ and for a subtree $T^{\prime}$ of $T$, by the notation $\left(T^{\prime}, \mathbf{S}^{\prime}\right) \preceq(T, \mathbf{S})$ we will mean that $\left(T^{\prime}, S_{1}^{\prime}, \ldots, S_{d}^{\prime}\right)$ is a regular vector subtree of $\left(T, S_{1}, \ldots, S_{d}\right)$. Finally, since we will deal exclusively with dyadic trees, we will omit the word "dyadic" in front of the words tree, vector tree, subtree and vector subtree.

Roughly speaking, the idea for the proof of Theorem 2 is to construct an appropriate regular vector subtree $(R, \mathbf{Q})$ of $(T, \mathbf{S})$ such that the coloring of $\otimes\left(T^{(2)}, \mathbf{S}\right)$ is reduced to a corresponding coloring of $\otimes(R, \mathbf{Q})$. Then, applying the HLLP Theorem, we will get the desired result.

Let us start with the following definition.

Definition 3. Let $T$ be a tree and let $\mathbf{S}=\left(S_{1}, \ldots, S_{d}\right)$ be a $d$-vector tree. We define the map

$$
\Lambda_{(T, \mathbf{S})}: \otimes\left(T^{(2)}, \mathbf{S}\right) \mapsto \otimes(T, \mathbf{S})
$$

as follows:

Let $\mathbf{t}=\left(t_{1}, t_{2}, s_{1}, \ldots, s_{d}\right) \in \otimes\left(T^{(2)}, \mathbf{S}\right)$. We set

$$
t^{\prime}=t_{1} \wedge_{T} t_{2}
$$

If $\left|t^{\prime}\right|_{T}=n$ is the order of $t^{\prime}$ in $T$, then for each $i=1, \ldots, d$ we set

$$
s_{i}^{\prime}=\operatorname{Pred}\left(s_{i}, S_{i}\right) \cap S_{i}(n) .
$$

Then we define

$$
\Lambda_{(T, \mathbf{S})}(\mathbf{t})=\left(t^{\prime}, s_{1}^{\prime}, \ldots, s_{d}^{\prime}\right) .
$$

It is easy to see that $\Lambda_{(T, \mathbf{S})}$ is an onto $\otimes(T, \mathbf{S})$ map.

As it has already been mentioned, if $R$ is a strong subtree of $T$, then for every $r_{1}, r_{2} \in R, r_{1} \wedge_{R} r_{2}=r_{1} \wedge_{T} r_{2}$. The next lemma plays a key role in the sequel and its proof is a straightforward consequence of the above-mentioned remark.

Lemma 4. Let $(R, \mathbf{Q})$ be a regular vector subtree of $(T, \mathbf{S})$ such that $R$ is a strong subtree of $T$. Then for all $\mathbf{t} \in \otimes\left(R^{(2)}, \mathbf{Q}\right)$,

$$
\Lambda_{(T, \mathbf{S})}(\mathbf{t})=\Lambda_{(R, \mathbf{Q})}(\mathbf{t}) .
$$

In the following let $(T, \mathbf{S})$, where $\mathbf{S}=\left(S_{1}, \ldots, S_{d}\right)$, be as stated in Theorem 2 and let $f: \otimes\left(T^{(2)}, \mathbf{S}\right) \rightarrow\{1, \ldots, r\}$ denote the finite coloring of $\otimes\left(T^{(2)}, \mathbf{S}\right)$. Let us call a regular vector subtree $(R, \mathbf{Q})$ of $(T, \mathbf{S})$ a reduced vector subtree of $(T, \mathbf{S})$, if $R$ is a strong subtree of $T$ and also for all $\mathbf{t}_{\mathbf{1}}, \mathbf{t}_{\mathbf{2}} \in \otimes\left(R^{(2)}, \mathbf{Q}\right)$ such that $\Lambda_{(R, \mathbf{Q})}\left(\mathbf{t}_{\mathbf{1}}\right)=$ $\Lambda_{(R, \mathbf{Q})}\left(\mathbf{t}_{\mathbf{2}}\right)$, the color of $\mathbf{t}_{\mathbf{1}}$ is the same as that of $\mathbf{t}_{\mathbf{2}}$. Formally, a regular vector subtree $(R, \mathbf{Q})$ of $(T, \mathbf{S})$ is called a reduced vector subtree of $(T, \mathbf{S})$ if $R$ is a strong subtree of $T$ and there is a coloring $g: \otimes(R, \mathbf{Q}) \rightarrow\{1, \ldots, r\}$ such that for all $\mathbf{t} \in \otimes\left(R^{(2)}, \mathbf{Q}\right)$,

$$
g\left(\Lambda_{(R, \mathbf{Q})}(\mathbf{t})\right)=f(\mathbf{t}) .
$$

In the next lemma it is shown that the existence of a reduced-vector subtree of $(T, \mathbf{S})$ easily yields Theorem 2 
Lemma 5. If $(R, \mathbf{Q})$ is a reduced subtree of $(T, \mathbf{S})$, then there exists a strong vector subtree $\left(T^{\prime}, \mathbf{S}^{\prime}\right)$ of $(R, \mathbf{Q})$ such that $\otimes\left(T^{\prime(2)}, \mathbf{S}^{\prime}\right)$ is monochromatic.

Proof. By the HLLP Theorem there is a strong vector subtree $\left(T^{\prime}, \mathbf{S}^{\prime}\right)$ of $(R, \mathbf{Q})$ and $c \in\{1, \ldots, r\}$ such that for all $\left(t^{\prime}, s_{1}^{\prime}, \ldots, s_{d}^{\prime}\right) \in \otimes\left(T^{\prime}, \mathbf{S}^{\prime}\right)$ we have that $g\left(t^{\prime}, s_{1}^{\prime}, \ldots, s_{d}^{\prime}\right)$ $=c$. Let $\mathbf{t} \in \otimes\left(T^{\prime(2)}, \mathbf{S}^{\prime}\right)$. Then by Lemma $4, \Lambda_{(R, \mathbf{Q})}(\mathbf{t})=\Lambda_{\left(T^{\prime}, \mathbf{S}^{\prime}\right)}(\mathbf{t})$. Therefore by the definition of the reduced vector subtree

$$
f(\mathbf{t})=g\left(\Lambda_{(R, \mathbf{Q})}(\mathbf{t})\right)=g\left(\Lambda_{\left(T^{\prime}, \mathbf{S}^{\prime}\right)}(\mathbf{t})\right)=c,
$$

and the proof is complete.

Hence our aim from now on is to find a reduced-vector subtree of $(T, \mathbf{S})$. This will be constructed by induction. For this purpose we make the following definition.

Definition 6. Assume that $\left(T^{\prime}, \mathbf{S}^{\prime}\right)$ is a regular vector subtree of $(T, \mathbf{S})$, where $\mathbf{S}^{\prime}=\left(S_{1}^{\prime}, \ldots, S_{d}^{\prime}\right)$ and $k \in \mathbb{N}$. Then a regular vector subtree $(R, \mathbf{Q})$ of $\left(T^{\prime}, \mathbf{S}^{\prime}\right)$ will be called a $k$-reduced vector subtree of $\left(T^{\prime}, \mathbf{S}^{\prime}\right)$ if it satisfies the following:

(i) The tree $R$ is a strong subtree of $T^{\prime}$.

(ii) For all $0 \leq m \leq k, R(m)=T^{\prime}(m)$ and $Q_{i}(m)=S_{i}^{\prime}(m)$ for all $1 \leq i \leq d$.

(iii) There is a coloring

$$
g_{(R, \mathbf{Q})}^{k}: T^{\prime}(k) \times S_{1}^{\prime}(k) \times \cdots \times S_{d}^{\prime}(k) \rightarrow\{1, \ldots, r\}
$$

such that for all $\mathbf{t} \in \otimes\left(R^{(2)}, \mathbf{Q}\right)$ with

$$
\Lambda_{(R, \mathbf{Q})}(\mathbf{t}) \in T^{\prime}(k) \times S_{1}^{\prime}(k) \times \cdots \times S_{d}^{\prime}(k),
$$

we have that

$$
g_{(R, \mathbf{Q})}^{k}\left(\Lambda_{(R, \mathbf{Q})}(\mathbf{t})\right)=f(\mathbf{t}) .
$$

Lemma 7. Suppose that for each $k \in \omega$ and each regular vector subtree $\left(T^{\prime}, S^{\prime}\right)$ of $(T, \mathbf{S})$ there exists a $k$-reduced vector subtree of $\left(T^{\prime}, \mathbf{S}^{\prime}\right)$. Then there also exists a reduced vector subtree $(R, \mathbf{Q})$ of $(T, \mathbf{S})$.

Proof. By our assumption, we construct a decreasing sequence $(T, \mathbf{S})=\left(R_{0}, \mathbf{Q}_{\mathbf{0}}\right) \succeq$ $\left(R_{1}, \mathbf{Q}_{\mathbf{1}}\right) \succeq \ldots$ of vector trees, with $\mathbf{Q}_{\mathbf{k}}=\left(Q_{1}^{k}, \ldots, Q_{d}^{k}\right)$, such that for each $k \geq 1$, $\left(R_{k}, \mathbf{Q}_{\mathbf{k}}\right)$ is a $(k-1)$-reduced vector subtree of $\left(R_{k-1}, \mathbf{Q}_{\mathbf{k}-\mathbf{1}}\right)$. We set $R=\bigcap_{k=1}^{\infty} R_{k}$ and for each $i=1, \ldots, d, Q_{i}=\bigcap_{k=1}^{n} Q_{i}^{k}$.

Let $\mathbf{Q}=\left(Q_{1}, \ldots, Q_{d}\right)$. It is easy to see that $R$ is a strong subtree of $T$ and $\mathbf{Q}$ is a regular vector subtree of $\mathbf{S}$. We claim that $(R, \mathbf{Q})$ is a reduced vector subtree of $(T, \mathbf{S})$. Indeed, let us set $g_{k}=g_{\left(R_{k}, \mathbf{Q}_{\mathbf{k}}\right)}^{k}, \Lambda_{k}=\Lambda_{\left(R_{k}, \mathbf{Q}_{\mathbf{k}}\right)}, g=\bigcup_{k=1}^{\infty} g_{k}$ and $\Lambda=\Lambda_{(R, \mathbf{Q})}$. Let $\mathbf{t} \in \otimes\left(R^{(2)}, \mathbf{Q}\right)$. Then there exists a unique $k_{0}$ such that $\Lambda_{(R, \mathbf{Q})}(\mathbf{t}) \in R\left(k_{0}\right) \times Q_{1}\left(k_{0}\right) \times \ldots \times Q_{d}\left(k_{0}\right)$. Since $(R, \mathbf{Q})$ is a regular vector subtree of $\left(R_{k_{0}}, \mathbf{Q}_{\mathbf{k}_{0}}\right)$ and $R$ is a strong subtree of $R_{k_{0}}$, by Lemma 4 we have that $\Lambda(\mathbf{t})=$ $\Lambda_{k_{0}}(\mathbf{t})$. Hence

and we are done.

$$
g(\Lambda(\mathbf{t}))=g_{k_{0}}\left(\Lambda_{k_{0}}(\mathbf{t})\right)=f(\mathbf{t})
$$

By the above lemma, it suffices to prove the existence of $k$-reduced subtrees of regular vector subtrees of $(T, \mathbf{S})$ for all $k$.

First let us treat the case $k=0$. 
Notation. Recall that if $T$ is a tree and $t \in T$, then $T_{t}=\left\{t^{\prime} \in T: t^{\prime} \geq t\right\}$. Generally, let $\mathbf{W}=\left(T_{1}, \ldots, T_{d}\right)$ be a $d$-vector tree and let $\left\{i_{1}<\cdots<i_{m}\right\} \subseteq\{1, \ldots, d\}$. Also let $\mathbf{t}=\left(t_{1}, \ldots, t_{m}\right) \in \otimes\left(T_{i_{1}}, \ldots, T_{i_{m}}\right)$. Then by $\mathbf{W}_{\mathbf{t}}$ we denote the $m$-vector tree $\left(T_{1}^{\prime}, \ldots, T_{m}^{\prime}\right)$, where for all $k=1, \ldots, m, T_{k}^{\prime}=\left(T_{i_{k}}\right)_{t_{k}}$.

Lemma 8. Let $\left(T^{\prime}, \mathbf{S}^{\prime}\right)$ be a regular vector subtree of $(T, \mathbf{S})$. Then there is a 0 reduced vector subtree $(R, \mathbf{Q})$ of $\left(T^{\prime}, \mathbf{S}^{\prime}\right)$.

Proof. Let $\mathbf{S}^{\prime}=\left(S_{1}^{\prime}, \ldots, S_{d}^{\prime}\right)$. Also let $t, s_{1}, \ldots, s_{d}$ be the roots of $T^{\prime}, S_{1}^{\prime}, \ldots, S_{d}^{\prime}$.

Let ImmSucc $\left(t, T^{\prime}\right)=\left\{t_{1}, t_{2}\right\}$. We set $T_{1}^{\prime}=\left(T^{\prime}\right)_{t_{1}}$ and $T_{2}^{\prime}=\left(T^{\prime}\right)_{t_{2}}$. Choose $s_{i}^{\prime} \in \operatorname{ImmSucc}\left(s_{i}, S_{i}\right)$, for $i=1, \ldots, d$ and let us set $S_{(i, i)}^{\prime}=\left(S_{i}^{\prime}\right)_{s_{i}^{\prime}}$. Then observe that

$$
\otimes\left(T_{1}^{\prime}, T_{2}^{\prime}, S_{(1,1)}^{\prime}, \ldots, S_{(d, d)}^{\prime}\right) \subseteq \otimes\left(T^{\prime(2)}, S_{1}^{\prime}, \ldots, S_{d}^{\prime}\right) .
$$

So by the HLLP Theorem there is a strong vector subtree $\left(T_{1}^{\prime \prime}, T_{2}^{\prime \prime}, S_{(1,1)}^{\prime \prime}, \ldots, S_{(d, d)}^{\prime \prime}\right)$ of $\left(T_{1}^{\prime}, T_{2}^{\prime}, S_{(1,1)}^{\prime}, \ldots, S_{(d, d)}^{\prime}\right)$ such that $\otimes\left(T_{1}^{\prime \prime}, T_{2}^{\prime \prime}, S_{(1,1)}^{\prime \prime}, \ldots, S_{(d, d)}^{\prime \prime}\right)$ is monochromatic. Let $t_{1}^{\prime}, t_{2}^{\prime}, s_{(1,1)}^{\prime}, \ldots, s_{(d, d)}^{\prime}$ be the roots of $T_{1}^{\prime \prime}, T_{2}^{\prime \prime}, S_{(1,1)}^{\prime \prime}, \ldots, S_{(d, d)}^{\prime \prime}$. We set $Q_{i}=$ $\left(S_{(i, i)}^{\prime \prime} \backslash\left\{s_{(i, i)}^{\prime}\right\}\right) \cup\left\{s_{i}\right\}$ and $\mathbf{Q}=\left(Q_{1}, \ldots, Q_{d}\right)$. To define the strong subtree $R$ of $T^{\prime}$ we proceed as follows: Let $t_{1}^{\prime \prime} \in \operatorname{ImmSucc}\left(t_{1}^{\prime}, T_{1}^{\prime \prime}\right), t_{2}^{\prime \prime} \in \operatorname{ImmSucc}\left(t_{2}^{\prime}, T_{2}^{\prime \prime}\right)$, and let us set $R_{1}=\left(T_{1}^{\prime \prime}\right)_{t_{1}^{\prime \prime}}, R_{1}=\left(T_{2}^{\prime \prime}\right)_{t_{2}^{\prime \prime}}$. Then we define $R=R_{1} \cup R_{2} \cup\{t\}$. It can be easily checked that $(R, \mathbf{Q})$ is a 0-reduced vector subtree of $\left(T^{\prime}, \mathbf{S}^{\prime}\right)$.

For the general case the following remark, which is an easy consequence of Lemma 4 will be very useful.

Remark. Suppose that $(R, \mathbf{Q})$ is a 0 -reduced vector subtree of $\left(T^{\prime}, \mathbf{S}^{\prime}\right)$ and let $\left(R^{\prime}, \mathbf{Q}^{\prime}\right)$ be a regular vector subtree of $(R, \mathbf{Q})$ satisfying the following conditions:

(i) $R^{\prime}$ is a strong subtree of $R$.

(ii) The roots of $R^{\prime}, Q_{1}^{\prime}, \ldots, Q_{d}^{\prime}$ coincide with the roots of $R, Q_{1}, \ldots, Q_{d}$.

Then $\left(R^{\prime}, \mathbf{Q}^{\prime}\right)$ is also an 0-reduced vector subtree of $\left(T^{\prime}, \mathbf{S}^{\prime}\right)$.

Lemma 9. Let $\left(T^{\prime}, \mathbf{S}^{\prime}\right)$ be a regular vector subtree of $(T, \mathbf{S})$ and let $k \in \omega$. Then there exists a $k$-reduced vector subtree $(R, \mathbf{Q})$ of $\left(T^{\prime}, \mathbf{S}^{\prime}\right)$.

Proof. The case $k=0$ has already been treated in the previous lemma. For reasons of notational simplicity we give the proof only for the case $k=1$. The procedure for the construction of a $k$-reduced vector subtree for an arbitrary $k>1$ follows from similar arguments.

Assume that $\left(T^{\prime}, \mathbf{S}^{\prime}\right)=\left(T^{\prime}, S_{1}^{\prime}, \ldots, S_{d}^{\prime}\right)$ is a regular vector subtree of $(T, \mathbf{S})=$ $\left(T, S_{1}, \ldots, S_{d}\right)$. Let $T^{\prime}(1)=\left\{t_{1}, t_{2}\right\}$ and for $i=1, \ldots, d$ let $S_{i}^{\prime}(1)=\left\{s_{(i, 1)}, s_{(i, 2)}\right\}$. We set $T_{1}=\left(T^{\prime}\right)_{t_{1}}, T_{2}=\left(T^{\prime}\right)_{t_{2}}, S_{(i, 1)}=\left(S_{i}^{\prime}\right)_{s_{(i, 1)}}$ and $S_{(i, 2)}=\left(S_{i}^{\prime}\right)_{s_{(i, 2)}}$ for $i=1, \ldots, d$. Let us define the $2 d+2$-vector tree:

$$
\mathbf{W}^{0}=\left(T_{1}, T_{2}, S_{(1,1)}, S_{(1,2)}, \ldots, S_{(d, 1)}, S_{(d, 2)}\right) .
$$

Let $\left\{\mathbf{r}_{1}, \ldots, \mathbf{r}_{2^{\mathbf{d}+1}}\right\}$ be an enumeration of $T^{\prime}(1) \times S_{1}^{\prime}(1) \times \cdots \times S_{d}^{\prime}(1)$.

By a finite induction we construct a decreasing sequence $\mathbf{W}^{0} \succeq \mathbf{W}^{1} \succeq \cdots \succeq$ $\mathbf{W}^{2^{d+1}}$ of regular $(2 d+2)$-vector subtrees such that for each $n=1, \ldots, 2^{d+1}$ the following property is satisfied: for all $1 \leq m \leq n$, the $d$-vector tree $\mathbf{W}_{\mathbf{r}_{m}}^{n}$ is a 0 reduced vector subtree of $\mathbf{W}_{\mathbf{r}_{m}}^{n-1}$ (for the definition of $\mathbf{W}_{\mathbf{r}_{m}}^{n}$ see the Notation above Lemma 8). 
The induction goes as follows. Suppose that $\mathbf{W}^{0}, \ldots, \mathbf{W}^{n}$ have been constructed so as to satisfy the required property. Let

$$
\mathbf{W}^{n}=\left(T_{1}^{n}, T_{2}^{n}, S_{(1,1)}^{n}, S_{(1,2)}^{n}, \ldots, S_{(d, 1)}^{n}, S_{(d, 2)}^{n}\right)
$$

and $\mathbf{r}_{\mathbf{n}+\mathbf{1}}=\left(t_{\varepsilon}, s_{\left(1, \varepsilon_{1}\right)}, \ldots, s_{\left(d, \varepsilon_{d}\right)}\right)$, where $\varepsilon, \varepsilon_{i} \in\{1,2\}$. By Lemma 8 there is a 0 -reduced vector subtree $\left(T_{\varepsilon}^{n+1}, S_{\left(1, \varepsilon_{1}\right)}^{n+1}, \ldots, S_{\left(d, \varepsilon_{d}\right)}^{n+1}\right)$ of

$$
\mathbf{W}_{\mathbf{r}_{n+1}}^{n}=\left(T_{\varepsilon}^{n}, S_{\left(1, \varepsilon_{1}\right)}^{n}, \ldots, S_{\left(d, \varepsilon_{d}\right)}^{n}\right) .
$$

Let $\varepsilon^{\prime}, \varepsilon_{i}^{\prime} \in\{1,2\}$ such that $\varepsilon^{\prime} \neq \varepsilon$ and $\varepsilon_{i}^{\prime} \neq \varepsilon_{i}$. Then we choose a strong vector subtree $\left(T_{\varepsilon^{\prime}}^{n+1}, S_{\left(1, \varepsilon_{1}^{\prime}\right)}^{n+1}, \ldots, S_{\left(d, \varepsilon_{d}^{\prime}\right)}^{n+1}\right)$ of $\left(T_{\varepsilon^{\prime}}^{n}, S_{\left(1, \varepsilon_{1}^{\prime}\right)}^{n}, \ldots, S_{\left(d, \varepsilon_{d}^{\prime}\right)}^{n}\right)$ compatible with $\left(T_{\varepsilon}^{n+1}, S_{\left(1, \varepsilon_{1}\right)}^{n+1}, \ldots, S_{\left(d, \varepsilon_{d}\right)}^{n+1}\right)$ in $\left(T^{\prime}, \mathbf{S}^{\prime}\right)$, and we set

$$
\mathbf{W}^{n+1}=\left(T_{1}^{n+1}, T_{2}^{n+1}, S_{(1,1)}^{n+1}, S_{(1,2)}^{n+1} \ldots, S_{(d, 1)}^{n+1}, S_{(d, 2)}^{n+1}\right) .
$$

By the remark after the case $k=0, \mathbf{W}_{\mathbf{r}_{m}}^{n+1}$ is a 0-reduced vector subtree of $\mathbf{W}_{\mathbf{r}_{m}}^{n}$ for all $m=1, \ldots, n+1$.

Let $\mathbf{W}^{2^{d+1}}=\left(T_{1}^{\prime}, T_{2}^{\prime}, S_{(1,1)}^{\prime}, S_{(1,2)}^{\prime} \ldots, S_{(d, 1)}^{\prime}, S_{(d, 2)}^{\prime}\right)$ be the final vector tree. We set $R=T^{\prime}(0) \cup T_{1}^{\prime} \cup T_{2}^{\prime}, Q_{i}=S_{i}^{\prime}(0) \cup S_{(i, 1)}^{\prime} \cup S_{(i, 2)}^{\prime}$ for $i=1, \ldots, d$. It is easily checked that $(R, \mathbf{Q})$ is a 1-reduced vector subtree of $\left(T^{\prime}, \mathbf{S}^{\prime}\right)$.

By the above lemmas the proof of Theorem 2 is completed.

Combining the HLLP Theorem and Theorem 2 we can derive a unified perfect tree version of them as follows.

For $m \in \mathbb{N}, T_{0}, \ldots, T_{m}$ perfect trees, $i \in\{0, \ldots, m\}$ and $\varepsilon \in\{1,2\}$, define

$$
\otimes\left(T_{0}, \ldots, T_{i}^{(\varepsilon)}, \ldots, T_{m}\right)=\bigcup_{n} T_{0}(n) \times \cdots \times T_{i}^{(\varepsilon)}(n) \times \cdots \times T_{m}(n),
$$

where

$$
\begin{aligned}
& T_{i}^{(1)}(n)=T_{i}(n) \text { and } \\
& T_{i}^{(2)}(n)=\left\{\left\{t_{1}, t_{2}\right\}: t_{1} \neq t_{2} \text { and } t_{1}, t_{2} \in T_{i}(n)\right\} .
\end{aligned}
$$

Since every perfect vector tree contains regular dyadic vector subtree, by the HLLP Theorem and Theorem 2, we have the following:

Theorem 10. For every $m \in \mathbb{N}, T_{0}, \ldots, T_{m}$ perfect trees, $i \in\{0, \ldots, m\}, \varepsilon \in\{1,2\}$ and every finite coloring of $\otimes\left(T_{0}, \ldots, T_{i}^{(\varepsilon)}, \ldots, T_{m}\right)$ there exists a regular perfect vector subtree $\left(S_{0}, \ldots, S_{m}\right)$ of $\left(T_{0}, \ldots, T_{m}\right)$, such that $\otimes\left(S_{0}, \ldots, S_{i}^{(\varepsilon)}, \ldots, S_{m}\right)$ is monochromatic.

\section{On subtrees of the Cantor tree}

3.1. Definitions and notations. By $C=\{0,1\}^{<\mathbb{N}}$ we denote the Cantor tree which is the set containing all finite sequences $t=(t(0), \ldots t(n)), n \in \mathbb{N}$, of 0 's and 1's including the empty sequence and partially ordered by the initial segment ordering denoted by $\leq$.

By $\prec$ we denote the lexicographical ordering of $C$, i.e. $t \prec t^{\prime}$ iff either $t<$ $t^{\prime}$ or $t$ and $t^{\prime}$ are incomparable and $t\left(n_{0}\right)<t^{\prime}\left(n_{0}\right)$, where $n_{0}=\min \{n \in \mathbb{N}$ : $t(n), t^{\prime}(n)$ are both defined and $\left.t(n) \neq t^{\prime}(n)\right\}$. The order of a $t \in C$ will be denoted 
by $|t|$ instead of $|t|_{C}$, and for every subtree $A$ of $C$ we denote by $L(A)$ the level set of $A$ in $C$. Also, by $t_{1} \wedge t_{2}$ we denote the infimum of $\left\{t_{1}, t_{2}\right\}$ in $C$. A downwards closed subtree $T$ of $C$ is a subset of $C$ such that for every $t \in T$ and every $s \in C$ with $s<t, s \in T$.

Definitions 11. Let $A$ be a subtree of $C$. Then

(a) The envelope $\hat{A}$ of $A$ in $C$ is defined to be the set

$$
\hat{A}=\bigcap\{T: A \subseteq T \text { and } T \text { is a downwards closed subtree of } C\} .
$$

(b) The regular envelope $\bar{A}$ of $A$ is defined to be the set

$$
\bar{A}=\bigcap\{R: A \subseteq R \text { and } R \text { is a regular subtree of } C\} .
$$

Note that for every subtree $A$ of $C, \bar{A}=\left.\hat{A}\right|_{L(A)}=\bigcup_{n \in L(A)} \hat{A}(n)$.

For $A, B$ non-empty subtrees of $C$ a map $\phi: A \rightarrow B$ is called a strong isomorphism of $A$ onto $B$ iff it is bijection and preserves both $<$ and $\prec$. If such a map exists we call $A, B$ strongly isomorphic subtrees of $C$ and we write $A \sim B$.

In the sequel we are mainly concerned with subtrees of $C$ that are finitely branching, pruned and rooted. So, by the term subtree we will always mean a subtree of $C$ with the above properties, unless otherwise stated.

It follows readily that if $T, S$ are strongly isomorphic subtrees of $C$, then there exists a unique strong isomorphism $\phi: T \rightarrow S$.

In order to describe the strongly isomorphic classes of subtrees of $C$ we introduce the following.

Definitions 12. Let $T$ be a subtree of $C$. Then the coding of $T$ is the function $f_{T}: \mathbb{N} \rightarrow \mathbb{N}^{<\mathbb{N}}$ defined as follows:

Let $n \in \mathbb{N}$ and $t_{0} \prec \cdots \prec t_{k}$ be the lexicographical ordering of $T(n)$. Then we set $f_{T}(n)=\left(m_{0}, \ldots, m_{k}\right)$, where $m_{i}=\left|\operatorname{ImmSucc}\left(t_{i}, T\right)\right|$.

Every function $f: \mathbb{N} \rightarrow \mathbb{N}<\mathbb{N}$ such that there exists a subtree $T$ of $C$ such that $f=f_{T}$, is called a tree coding.

A coding of a skew subtree of $C$ is called a skew tree coding and of a perfect subtree, a perfect tree coding. If $f$ is a tree coding, then every subtree of coding $f$ will be called an $f$-subtree of $C$.

We also use another function related to a subtree $T$ of $C$. To define it we need some additional notation. Let $T$ be a subtree of $C$. For $t, t^{\prime} \in T$ we define $t \ll t^{\prime}$ iff either $|t|_{T}<\left|t^{\prime}\right|_{T}$ or $|t|_{T}=\left|t^{\prime}\right|_{T}$ and $t \prec t^{\prime}$. It follows easily that $(T, \ll)$ is well ordered, isomorphic to $\mathbb{N}$ and therefore $T$ takes the form of a sequence $T=\left(t_{n}\right)_{n}$, $n \in \mathbb{N}$, which will be called the natural expansion of $T$. Note that if $T=\left(t_{n}\right)_{n}$ and $S=\left(s_{n}\right)_{n}$ are isomorphic subtrees of $T$, then the unique strong isomorphism $\phi: T \rightarrow S$ is given by $\phi\left(t_{n}\right)=s_{n}$ for all $n$.

Notation. Let $T=\left(t_{n}\right)_{n}$ be a subtree of $C$.

(a) For each $k \in \mathbb{N}$, we set $L_{k}^{T}=\left\{n \in \mathbb{N}: t_{n} \in T(k)\right\}$.

(b) For each $i \in \mathbb{N}$, we set $I_{i}^{T}=\left\{n \in \mathbb{N}: t_{n} \in \operatorname{ImmSucc}\left(t_{i}, T\right)\right\}$.

Observe that for every subtree $T$ of $C, L_{0}^{T}=\{0\}$ and also that

(a) $\mathbb{N}=\bigcup_{k} L_{k}^{T}$ and $L_{k}^{T}, L_{k+1}^{T}$ are successive finite intervals of $\mathbb{N}$ for all $k$.

(b) For every $k>1, L_{k}^{T}=\bigcup\left\{I_{i}^{T}: i \in L_{k-1}^{T}\right\}$ and $I_{i}^{T}, I_{i+1}^{T}$ are also successive finite intervals of $\mathbb{N}$. 
Definition 13. Let $T$ be a subtree of $C$. We define $\pi_{T}: \mathbb{N} \backslash\{0\} \rightarrow \mathbb{N}$ by

$$
\pi_{T}(n)=i \text { iff } n \in I_{i}^{T}
$$

Note that for strongly isomorphic subtrees $S, T$ of $C$ we have that $\pi_{T}=\pi_{S}$.

\subsection{The family of rapidly increasing subtrees of $C$.}

Definition 14. Let $T=\left(t_{n}\right)_{n}$ be a subtree of $C$.

(a) We call $T$ an increasing subtree of $C$ if for all $n,\left|t_{n}\right|<\left|t_{n+1}\right|$, where $\left|t_{n}\right|$ is the order of $t_{n}$ in $C$.

(b) We call $T$ a rapidly increasing subtree of $C$ if it is an increasing subtree of $C$ and it satisfies the following condition: For every $n$ if there exists an $n^{\prime}$ such that $t_{n+1}, t_{n+2} \in \operatorname{ImmSucc}\left(t_{n^{\prime}}, T\right)$, then $\left|t_{n}\right| \leq\left|t_{n+1} \wedge t_{n+2}\right|$, where $t_{n+1} \wedge t_{n+2}$ is the infimum of $t_{n+1}, t_{n+2}$ in $C$.

(c) We call $T$ a rapidly increasing $f$-subtree of $C$ if $T$ is a rapidly increasing subtree of $C$ of coding $f$. The set of all rapidly increasing $f$-subtrees of $C$ will be denoted by $C_{f}^{*}$.

The following proposition verifies that for every tree coding $f$ the family $C_{f}^{*}$ is perfectly dense.

Proposition 15. For every perfect subtree $S$ of $C$ and for every tree coding $f$, there exists a subtree $T$ of $S$ such that $T \in C_{f}^{*}$.

Proof. The proof is based on the following:

Claim: For every $s \in S$ and $n \in \mathbb{N}$ there exist $t_{0} \prec \cdots \prec t_{n}$ successors of $s$ in $S$, such that $\left|t_{i}\right| \leq\left|t_{i+1} \wedge t_{i+2}\right|$ for all $0 \leq i \leq n-2$.

Proof of the claim. For $n=0$, it is trivial. Suppose that the claim is true for some $n$ and every $s \in S$.

Let $s \in S$. Then since $S$ is a perfect tree there exist $s^{\prime} \prec s^{\prime \prime}$ successors of $s$ in $S$ such that $\left|s^{\prime \prime}\right|>\left|s^{\prime}\right|$. By the inductive hypothesis we can choose $t_{0}^{\prime} \prec \cdots \prec t_{n}^{\prime}$ successors of $s^{\prime \prime}$ in $S$ satisfying the conditions of the claim. We set $t_{0}=s^{\prime}$ and $t_{i}=t_{i-1}^{\prime}$ for $i=1, \ldots n+1$. Then clearly $t_{0} \prec \cdots \prec t_{n+1}$ and $\left|t_{i}\right| \leq\left|t_{i+1} \wedge t_{i+2}\right|$ for all $i \leq n-1$.

Using the above claim we construct by induction a rapidly increasing $f$-subtree $T$ of $S$ as follows:

We choose a $t_{0} \in S$ and we set $T(0)=\left\{t_{0}\right\}$. Suppose that we have constructed $T(k)=\left\{t_{0}^{k} \prec \cdots \prec t_{m}^{k}\right\}$ for some $k$ and let $f(k)=\left(n_{0}, \ldots, n_{m}\right)$. Then we choose a successor $s_{0}$ of $t_{0}^{k}$ in $S$ such that $\left|s_{0}\right|>\left|t_{m}^{k}\right|$ and we apply the above claim for $n=n_{0}-1$ and $s=s_{0}$. If $t_{0}^{0}, \ldots, t_{n_{0}-1}^{0}$ are the resulting nodes, then we choose the successor $s_{1}$ of $t_{1}^{k}$ in $S$ such that $\left|s_{1}\right|>\left|t_{n_{0}-1}^{0}\right|$, and we again apply the claim for $s=s_{1}$ and $n=n_{1}^{k}-1$. We continue in the obvious way and construct $T(k+1)$.

Note that if $T=\left(t_{n}\right)_{n}$ is an increasing subtree of $C$ and $\bar{T}$ its regular envelope, then $L(T)=L(\bar{T})=\left\{m_{n}=\left|t_{n}\right|: n \in \mathbb{N}\right\}$ and, for every $n, \bar{T}(n) \cap T=\left\{t_{n}\right\}$. In view of this remark we give the following definition.

Definition 16. Let $T=\left(t_{n}\right)_{n}$ be an increasing subtree of $C$ and let $\bar{T}$ be its regular envelope. We define the function $\sigma_{T}: \mathbb{N} \rightarrow \mathbb{N}$ as follows: Let $n \in \mathbb{N}$ and let $\bar{T}(n)=\left\{\bar{t}_{0} \prec \cdots \prec \overline{t_{k}}\right\}$ be the lexicographic ordering of $\bar{T}(n)$. Then $\sigma_{T}(n)$ is the unique $i \in\{0, \ldots, k\}$ such that $\overline{t_{i}}=t_{n}$. 
The following proposition is the first main result of this section.

Proposition 17. For every tree coding $f$ there exist a unique skew tree coding $\bar{f}$ and a unique function $\sigma_{f}: \mathbb{N} \rightarrow \mathbb{N}$ such that for every rapidly increasing $f$-subtree $T$ of $C, \bar{T}$ is a regular $\bar{f}$-subtree and $\sigma_{T}=\sigma_{f}$.

The proof of the above proposition is based on some lemmas which will be stated below. In all these lemmas we suppose that $T$ is a rapidly increasing $f$-subtree of $C,\left(t_{n}\right)_{n}$ is the natural expansion of $T$ and $L(T)=\left\{m_{n}=\left|t_{n}\right|: n \in \mathbb{N}\right\}$ is the level set of $T$ in $C$. We also set $\pi=\pi_{T}, I_{i}=I_{i}^{T}, i \in \mathbb{N}$, and $L_{k}=L_{k}^{T}, k \in \mathbb{N}$.

Notation. For all $t \in C, n<|t|$ we set $t\lceil n=(t(0), \ldots, t(n-1))$, i.e. $t \uparrow n=$ $\operatorname{Pred}(t, C) \cap C(n)$.

Lemma 18. Let $i \in \mathbb{N}$. Then for every non-empty interval $I \subseteq I_{i}$ and every $n<\min I$, the set $\left\{t_{m}\left\lceil m_{n}: m \in I\right\}\right.$ is a singleton.

Proof. For $|I|=1$ the lemma trivially holds, and for $|I|=2$ it follows easily from the definition of the rapidly increasing tree. So suppose that $\left|I_{i}\right| \geq 2$ and that the lemma is true for all $1 \leq|I| \leq k$, where $2 \leq k<\left|I_{i}\right|$. Let $I \subseteq I_{i}$, $|I|=k+1, I=\left\{n_{0}, \ldots, n_{0}+k\right\}$ for some $n_{0}>n$. We set $I_{0}=\left\{n_{0}, n_{0}+1\right\}$, $I_{1}=\left\{n_{0}+1, \ldots, n_{0}+k\right\}$. By the inductive hypothesis, $\left\{t_{m}\left\lceil m_{n}: m \in I_{0}\right\}=\left\{s_{0}\right\}\right.$ and $\left\{t_{m}\left\lceil m_{n}: m \in I_{1}\right\}=\left\{s_{1}\right\}\right.$. Since $I_{0} \cap I_{1}=\left\{n_{0}+1\right\}$, we have that $s_{0}=s_{1}=$ $t_{n_{0}+1}\left\lceil m_{n}\right.$. Therefore $\left\{t_{m}\left\lceil m_{n}: m \in I\right\}\right.$ is a singleton.

Lemma 19. Let $n, k \in \mathbb{N}$ such that $n \in L_{k}$.

(a) If $n=\max L_{k}$, then $\bar{T}(n)=\left\{s_{i}^{n}: i \in L_{k}\right\}$, where $s_{n}^{n}=t_{n}$, and for all $i \in L_{k}$, $i<n, s_{i}^{n}=t_{m}\left\lceil m_{n}\right.$ for all $m \in I_{i}$. Also for $i<j, s_{i}^{n} \prec s_{j}^{n}$.

(b) If $n<\max L_{k}$, then $\bar{T}(n)=\left\{s_{i}^{n}: i \in[\pi(n+1), n]\right\}$, where $s_{n}^{n}=t_{n}$ and for all $i \neq n, s_{i}^{n}=t_{m}\left\lceil m_{n}\right.$ for all $m \in I_{i}$ and $m>n$. Also if $n_{k}=\min L_{k}$, then

(i) If $i<j$ and $i, j \in\left[\pi(n+1), n_{k}\right)$, then $s_{i}^{n} \prec s_{j}^{n}$.

(ii) If $i<j$, and $i, j \in\left[n_{k}, n\right]$, then $s_{i}^{n} \prec s_{j}^{n}$.

(iii) If $i \in\left[n_{k}, n\right]$ and $j \in\left[\pi(n+1), n_{k}\right)$, then $s_{i}^{n} \prec s_{j}^{n}$.

Proof. (a) If $n=\max L_{k}$, then

$$
\begin{aligned}
\bar{T}(n) & =\left\{t_{m}\left\lceil m_{n}: m>n\right\}=\left\{t_{m}\left\lceil m_{n}: m \in L_{k+1}\right\}\right.\right. \\
& =\bigcup_{i \in L_{k}}\left\{t_{m}\left\lceil m_{n}: m \in I_{i}\right\} .\right.
\end{aligned}
$$

By Lemma 18, we have that $\left\{t_{m}\left\lceil m_{n}: m \in I_{i}\right\}\right.$ is a singleton and so we can set $s_{i}^{n}=t_{m}\left\lceil m_{n}\right.$ for all $m \in I_{i}, i \in L_{k}$. Clearly $s_{n}^{n}=t_{n}$ and $s_{i}^{n} \prec s_{j}^{n}$ for all $i<j$ in $L_{k}$.

(b) If $n<\max L_{k}$, then $k \geq 1, n+1 \in L_{k}$, so $\pi(n+1) \in L_{k-1}$. Note that $\bar{T}(n)=$ $\left\{t_{m}\left\lceil m_{n}: m \in\left[n+1, \max I_{n}\right]\right\}=\bigcup_{i \in[\pi(n+1), n]}\left\{t_{m}\left\lceil m_{n}: m \in I_{i}\right.\right.\right.$ and $\left.m>n\right\}$.

By Lemma 18 we can set $s_{i}^{n}=t_{m}\left\lceil m_{n}\right.$ for all $m \in I_{i}$ and $m>n$. Then $s_{n}^{n}=t_{n}$, and the verification of (i)-(iii) is easily checked.

Lemma 20. Let $n, k \in \mathbb{N}$ such that $n \in L_{k}$ and let $n_{k}=\min L_{k}$. Then:

(a) $|\bar{T}(n)|=n-\pi(n+1)+1, \bar{T}(n)=\left\{s_{i}^{n}: i \in[\pi(n+1), n]\right\}$, where $s_{n}^{n}=t_{n}$ and for $i \neq n, s_{i}^{n}=t_{m}\left\lceil m_{n}\right.$ for all $m \in I_{i}$ and $m>n$.

(b) The function $\sigma_{T}: \mathbb{N} \rightarrow \mathbb{N}$, is given by $\sigma_{T}(n)=n-n_{k}$. 
Proof. The proof follows easily from Lemma 19] It only suffices to observe that if $n=\max L_{k}$, then $\pi(n+1)=n_{k}$.

Lemma 21. The coding $f_{\bar{T}}$ of the regular envelope $\bar{T}$ of $T$ is given by

$$
f_{\bar{T}}(n)=\left(\varepsilon_{0}^{n}, \ldots, \varepsilon_{n-\pi(n+1)}^{n}\right),
$$

where

(a) if $\pi(n+1) \neq \pi(n+2)$, then $\varepsilon_{i}^{n}=1$ for all $i=0, \ldots, n-\pi(n+1)$, and

(b) if $\pi(n+1)=\pi(n+2)$, then $\sigma_{T}(n+1) \leq n-\pi(n+1), \varepsilon_{\sigma_{T}(n+1)}=2$ and $\varepsilon_{i}^{n}=1$ for all $i=0, \ldots, n-\pi(n+1)$.

Proof. By Lemma 20, $|\bar{T}(n)|=n-\pi(n+1)+1$ and so $f_{\bar{T}}(n)=\left(\varepsilon_{0}^{n}, \ldots, \varepsilon_{n-\pi(n+1)}^{n}\right)$.

(a) If $\pi(n+1) \neq \pi(n+2)$, then $|\bar{T}(n)|=|\bar{T}(n+1)|$ and so $\varepsilon_{i}^{n}=1$ for all $i=0, \ldots, n-\pi(n+1)$.

(b) If $\pi(n+1)=\pi(n+2)$, then $|\bar{T}(n+1)|=|\bar{T}(n)|+1$ and so there exists a unique $i_{0} \in\{0, \ldots, n-\pi(n+1)\}$ such that $\varepsilon_{i_{0}}^{n}=2$ and $\varepsilon_{i}^{n}=1$ for all $i \neq i_{0}$. Let $\bar{T}(n)=\left\{t_{0}^{n} \prec \cdots \prec t_{n-\pi(n+1)}^{n}\right\}$ and $\bar{T}(n+1)=\left\{t_{0}^{n+1} \prec \cdots \prec t_{n-\pi(n+1)+1}^{n+1}\right\}$. Note that $i_{0}$ is the unique $i$ such that

$$
t_{i}^{n}=t_{i}^{n+1}\left\lceil m_{n}=t_{i+1}^{n+1} \uparrow m_{n} .\right.
$$

Since $\pi(n+1)=\pi(n+2)$ and $T$ is rapidly increasing, we get that $t_{n+1}\left\lceil m_{n}=\right.$ $t_{n+2}\left\lceil m_{n}\right.$. Also $t_{n+1}, t_{n+2}$ belong to the same level of $T$ and so by Lemma 19(b), we have that $t_{n+1}=t_{\sigma_{T}(n+1)}^{n+1}$ and $t_{n+2}\left\lceil m_{n+1}=t_{\sigma_{T}(n+1)+1}^{n+1}\right.$. Hence

$$
t_{\sigma_{T}(n+1)}^{n+1}\left\lceil m_{n}=t_{\sigma_{T}(n+1)+1}^{n+1}\left\lceil m_{n}\right.\right.
$$

and therefore $i_{0}=\sigma_{T}(n+1)$.

Proof of Proposition 17. By Lemmas 20 and 21, we have that $f_{\bar{T}}, \sigma_{T}$ are independent to the choice of the rapidly increasing $f$-subtree $T$. Hence we can set $\bar{f}=f_{\bar{T}}$ and $\sigma_{f}=\sigma_{T}$. The proof of Proposition 17 is completed.

Definition 22. The function $\bar{f}$ and $\sigma_{f}$ satisfying the conclusion of Proposition 17 will be called the regular envelope and the level parametrization of $f$, respectively.

We will also show that for every regular $\bar{f}$-subtree $R$ of $C$, there exists a unique $R^{\prime} \in C_{f}^{*}$ such that $\overline{R^{\prime}}=R$. For this we will need the following notation.

Notation. Let $f$ be a tree coding. By $C_{f}$ we denote the set of all regular $f$-subtrees of $C$. Let $\sigma_{f}$ be the level parametrization of $f$ and $R \in C_{f}$. For each $n$, let $R(n)=\left\{t_{0}^{n} \prec \cdots \prec t_{k_{n}}^{n}\right\}$ be the lexicographic ordering of $R(n)$. Then we set

$$
\sigma_{f}(R)=\left\{t_{\sigma_{f}(n)}^{n}: n \in \mathbb{N}\right\} .
$$

Proposition 23. Let $f$ be a tree coding with regular envelope $\bar{f}$ and level parametrization $\sigma_{f}$. Then for every $R \in C_{\bar{f}}, \sigma_{f}(R)$ is a rapidly increasing $f$-subtree of $C$, such that $\overline{\sigma_{f}(R)}=R$. Moreover, $\sigma_{f}(R)$ is the unique rapidly increasing $f$-subtree of $C$ with the above property.

Proof. Let $T \in C_{f}^{*}, \bar{T}$ be its regular envelope and let $\phi: \bar{T} \rightarrow R$ be the unique strong isomorphism from $\bar{T}$ onto $R$. As has already been indicated, if $\bar{T}=\left(\bar{t}_{n}\right)_{n}$ and $R=\left(r_{n}\right)_{n}$ are the natural enumerations of $\bar{T}, R$ we have that $\phi\left(\overline{t_{n}}\right)=r_{n}$ for all $n$. We set $\sigma_{f}(R)=R^{\prime}$ and we will show that $R^{\prime} \in C_{f}^{*}$ and $\overline{R^{\prime}}=R$. 
First observe that $\phi(T)=R^{\prime}$ and so $R^{\prime}$ and $T$ are strongly isomorphic. Therefore $R^{\prime}$ is an $f$-subtree of $C$. Let $T=\left(t_{n}\right)_{n} R^{\prime}=\left(r_{n}^{\prime}\right)_{n}$ be the natural enumerations of $T$ and $R^{\prime}$. Then $\phi\left(t_{n}\right)=r_{n}^{\prime}$ for all $n$ and so $\left|t_{n}\right|_{\bar{T}}=\left|r_{n}^{\prime}\right|_{R}=n$. Since $R$ is a regular subtree of $T$, we have that $\left|r_{n}^{\prime}\right|<\left|r_{n+1}^{\prime}\right|$ for all $n$ and so $R^{\prime}$ is an increasing subtree of $C$. To show that $R^{\prime}$ is also rapidly increasing, suppose that $r_{n+1}^{\prime}, r_{n+2}^{\prime} \in \operatorname{ImmSucc}\left(r_{n^{\prime}}^{\prime}, R^{\prime}\right)$ for some $n^{\prime} \leq n$. Then $t_{n+1}, t_{n+2} \in \operatorname{ImmSucc}\left(t_{n^{\prime}}, T\right)$ and since $T \in C_{f}^{*}$, there exists a $\bar{t} \in \bar{T}$ such that $|\bar{t}|_{T}=\left|t_{n}\right|_{T}$ and $\bar{t}=t_{n+1} \wedge_{\bar{T}} t_{n+2}$. So if $r=\phi(\bar{t})$, then $|r|_{R}=\left|r_{n}\right|_{R}$ and $r=r_{n+1}^{\prime} \wedge_{R} r_{n+2}^{\prime}$. Since $R$ is a regular subtree of $C$, we have that $|r|=\left|r_{n}\right|$ and $r \leq r_{n+1}^{\prime} \wedge r_{n+2}^{\prime}$. Hence $\left|r_{n}\right| \leq\left|r_{n+1}^{\prime} \wedge r_{n+2}^{\prime}\right|$.

To show that $\overline{R^{\prime}}=R$ observe that $L\left(R^{\prime}\right)=L(R)$ since $L(T)=L(\bar{T})$. Also, since $T$ dominates $\bar{T}$ we have that $R^{\prime}$ dominates $R$ (that is, for every $r \in R$ there exists an $r^{\prime} \in R^{\prime}$ such that $\left.r \leq r^{\prime}\right)$. Therefore, $R=\left.\hat{R}\right|_{L(R)}=\left.\hat{R}^{\prime}\right|_{L\left(R^{\prime}\right)}=\overline{R^{\prime}}$. Finally, if $R^{\prime \prime} \in C_{f}^{*}$ with $\overline{R^{\prime \prime}}=R$, then $\sigma_{R^{\prime \prime}}=\sigma_{f}$ and hence $R^{\prime \prime}=R^{\prime}$.

Propositions 23 and 17 readily yield the following, which is one of the main ingredients of the proof of Theorem A.

Proposition 24. The families $C_{f}^{*}$ and $C_{\bar{f}}$ are homeomorphic subspaces of the compact space $2^{C}$ under the map $T \rightarrow \bar{T}$, with inverse $R \rightarrow \sigma_{f}(R)$.

\section{Abstract Ramsey theory}

In this section we will present briefly and without proof an abstract Ramsey theory due to $\mathrm{S}$. Todorčević ([T2]). We consider two sets $\mathcal{R}, \mathcal{S}$ and two binary relations $\leq \subseteq \mathcal{S}^{2}, \leq^{0} \subseteq \mathcal{R} \times \mathcal{S}$ such that

(a) $\leq$ is a quasi ordering (i.e. reflexive and transitive relation).

(b) For every $A \in \mathcal{R}$ and $S, T \in \mathcal{S}$, if $A \leq^{0} S$ and $S \leq T$, then $A \leq^{0} T$.

The sets $\mathcal{R}, \mathcal{S}$ are equipped with approximations $p=\left(p_{n}\right)_{n}, q=\left(q_{n}\right)_{n}$, respectively, where every $p_{n}$ (resp. $q_{n}$ ) is a function with domain $\mathcal{R}$ (resp. $\mathcal{S}$ ). We denote by $\mathcal{P}_{n}, \mathcal{Q}_{n}$ the ranges of $p_{n}, q_{n}$ respectively, and we set $\mathcal{P}=\bigcup_{n} \mathcal{P}_{n}, \mathcal{Q}=\bigcup_{n} \mathcal{Q}_{n}$.

For every $A \in \mathcal{R}$ (respectively $T \in \mathcal{S}$ ) we call $p_{n}(A)$ (respectively $q_{n}(T)$ ) the $n^{\text {th }}$-approximation to $A$ (resp. to $T$ ). The first three properties which we assume for the sequences of functions $\left(p_{n}\right)_{n},\left(q_{n}\right)_{n}$ are the following:

\section{Axiom 1.}

(a) $p_{0}(A)=p_{0}(B)$ for all $A, B \in \mathcal{R}$.

(b) $q_{0}(S)=q_{0}(T)$ for all $S, T \in \mathcal{S}$.

\section{Axiom 2.}

(a) For all $A, B \in \mathcal{R}, A \neq B$ implies $p_{d}(A) \neq p_{d}(B)$ for some $d$.

(b) For all $S, T \in \mathcal{S}, S \neq T$ implies $q_{n}(S) \neq q_{n}(T)$ for some $n$.

\section{Axiom 3.}

(a) For all $A, B \in \mathcal{R}$ and all $d, e, p_{d}(A)=p_{e}(B)$ implies $d=e$ and $p_{i}(A)=p_{i}(B)$ for all $i \leq d$.

(b) For all $S, T \in \mathcal{S}$ and all $n, m, q_{n}(S)=q_{m}(T)$ implies $m=n$ and $q_{i}(S)=$ $q_{i}(T)$ for all $i \leq n$.

We consider $\mathcal{P}, \mathcal{Q}$ as topological spaces with the discrete topology and $\mathcal{P}^{\mathbb{N}}, \mathcal{Q}^{\mathbb{N}}$ with the Tychonoff (product) topology. Every element $A$ of $\mathcal{R}$ (resp. $T$ of $\mathcal{S}$ ) is identified with the sequence $\left(p_{n}(A)\right)_{n} \in \mathcal{P}^{\mathbb{N}}\left(\operatorname{resp}\left(p_{n}(T)\right)_{n} \in \mathcal{Q}^{\mathbb{N}}\right)$ and so $\mathcal{R}, \mathcal{S}$ can be considered as subspaces of the topological spaces $\mathcal{P}^{\mathbb{N}}, \mathcal{Q}^{\mathbb{N}}$. 
Definition 25. We say that $\leq$ admits a finitization if there is a relation $\leq_{\text {fin }}$ on $\mathcal{Q}$ such that for all $S, T \in \mathcal{S}$,

$$
S \leq T \quad \text { iff } \quad \forall n \exists m \quad q_{n}(S) \leq_{\text {fin }} q_{m}(T) .
$$

Definition 26. For any $a, b \in \mathcal{P}$ we say that $b$ end-extends $a$, and we write $a \unlhd b$ if there exists $A \in \mathcal{R}$ and $m \leq n$ such that $a=p_{m}(A)$ and $b=p_{n}(A)$.

Definition 27. We say that $\leq^{0}$ admits a finitization if there is a relation $\leq_{\text {fin }}^{0}$ of $\mathcal{P} \times \mathcal{Q}$ such that for all $A \in \mathcal{R}, T \in \mathcal{S}$,

$$
A \leq^{0} T \text { iff } \forall n \exists m \quad p_{n}(A) \leq_{\text {fin }}^{0} q_{m}(T),
$$

and for all $a, b \in \mathcal{P}$ and $u \in \mathcal{Q}$,

$$
a \unlhd b \text { and } b \leq_{\text {fin }}^{0} u \text { implies } a \leq_{\text {fin }}^{0} u .
$$

Axiom 4. The relations $\leq^{0}, \leq$ admit finitizations $\leq_{\text {fin }}^{0}, \leq_{\text {fin }}$ respectively, and moreover for every $u \in \mathcal{Q}$ they have the property that the collections

$$
\begin{aligned}
& \left\{a \in \mathcal{P}: a \leq_{\text {fin }}^{0} u\right\}, \\
& \left\{v \in \mathcal{Q}: v \leq_{\text {fin }} u\right\}
\end{aligned}
$$

are both finite.

Definition 28. For $a \in \mathcal{P}$ and $T \in \mathcal{S}$ we define $\operatorname{depth}_{T}(a)=\min \left\{k: a \leq_{\text {fin }}^{0} q_{k}(T)\right\}$ if $a \leq_{\text {fin }}^{0} q_{k}(T)$ for some $k$, and $\operatorname{depth}_{T}(a)=-1$ otherwise.

Definition 29. (a) Let $a \in \mathcal{P}, u \in \mathcal{Q}$ and $T \in \mathcal{S}$. Then we define

$$
\begin{gathered}
{[a, T]=\left\{A \in \mathcal{R}: \exists d p_{d}(A)=a \text { and } A \leq^{0} T\right\},} \\
{[u, T]=\left\{S \in \mathcal{S}: \exists n q_{n}(S)=u \text { and } S \leq T\right\},} \\
{[a]=\left\{A \in \mathcal{R}: \exists d p_{d}(A)=a\right\} .}
\end{gathered}
$$

The sets $[a, T]$ will be referred to as basic sets.

(b) For $n \in \mathbb{N}$ and $T \in \mathcal{S}$ we define

$$
[n, T]=\left[q_{n}(T), T\right] .
$$

Note. The basic sets $[a, T]$ do not necessarily form a base for a topology. Hence they are not referred to as basic open sets. Let us also note that as it follows from Axiom 4, each basic set $[a, T]$ is closed in $\mathcal{P}^{\mathbb{N}}$. Moreover the sets $[a], a \in \mathcal{P}$ form a clopen basis for the topology of $\mathcal{R}$.

\section{Axiom 5.}

(i) For all $a \in \mathcal{P}$ and $T \in \mathcal{S}$, if $[a, T] \neq \emptyset$ and $S \in\left[\operatorname{depth}_{T}(a), T\right]$, then $[a, S] \neq \emptyset$.

(ii) For all $a \in \mathcal{P}$ and $S, T \in \mathcal{S}$, if $[a, T] \neq \emptyset, S \leq T$ and $[a, S] \neq \emptyset$, then there exists $T^{\prime} \in\left[\operatorname{depth}_{T}(a), T\right]$ such that $\left[a, T^{\prime}\right] \subseteq[a, S]$.

Definition 30. A subset $\mathcal{X}$ of $\mathcal{R}$ is $\mathcal{S}$-Ramsey if for every basic set $[a, T] \neq \emptyset$ there is an $S \in\left[\operatorname{depth}_{T}(a), T\right]$ such that either

(i) $[a, S] \subseteq \mathcal{X}, \quad$ or

(ii) $[a, S] \subseteq \mathcal{X}^{c}$.

If the second alternative always holds, then we say that $\mathcal{X}$ is $\mathcal{S}$-Ramsey null. 
Definition 31. A subset $\mathcal{X}$ of $\mathcal{R}$ is $\mathcal{S}$-Baire if for every basic set $[a, T] \neq \emptyset$ there is a $b \in \mathcal{P}$ end-extending $a$ and an $S \in \mathcal{S}$ with $S \leq T$, such that $[b, S] \neq \emptyset$ and either

(i) $[b, S] \subseteq \mathcal{X}, \quad$ or

(ii) $[b, S] \subseteq \mathcal{X}^{c}$.

If the second alternative always holds, then we say that $\mathcal{X}$ is $\mathcal{S}$-meager.

Note. It easy to see that the $\mathcal{S}$-Ramsey and $\mathcal{S}$-Baire sets form algebras of sets, and the $\mathcal{S}$-Ramsey null and $\mathcal{S}$-meager sets form ideals of the corresponding algebras.

The most crucial Axiom of the theory is the following.

Axiom 6. For all $d \in \mathbb{N}$, given $\mathcal{Y} \subseteq \mathcal{P}_{d+1}$, for all $a \in \mathcal{P}$ and $T \in \mathcal{S}$, if $[a, T] \neq \emptyset$, then there exists $S \in\left[\operatorname{depth}_{T}(a), T\right]$ such that either

(a) $p_{d+1}^{\prime \prime}[a, S] \subseteq \mathcal{Y} \quad$ or

(b) $p_{d+1}^{\prime \prime}[a, S] \subseteq \mathcal{Y}^{c}$.

Definition 32. A Souslin scheme on a set $X$ is a family $\left(F_{s}\right)_{s \in \mathbb{N}<\mathbb{N}}$, indexed by $\mathbb{N}<\mathbb{N}$. The Souslin operator $\mathcal{A}$ applied to such a scheme produces the set

$$
\mathcal{A}\left(\left(F_{s}\right)_{s}\right)=\bigcup_{x \in \mathbb{N}^{\mathbb{N}}} \bigcap_{n} F_{x \mid n}
$$

A family $\mathfrak{F}$ of subsets of $X$ is said to be closed under the Souslin operation $\mathcal{A}$ if for every Souslin scheme $\left(F_{s}\right)_{s}$ of elements of $\mathfrak{F}$,

$$
\mathcal{A}\left(\left(F_{s}\right)_{s}\right) \in \mathfrak{F} \text {. }
$$

The following theorem is the main result of the abstract Ramsey theory.

Theorem 33. Suppose that $\left(\mathcal{R}, \mathcal{S}, \leq, \leq^{0}, p, q\right)$ satisfies Axioms 1-6 and that $\mathcal{S}$ is closed as a subset of $\mathcal{Q}^{\mathbb{N}}$. Then the algebras of $\mathcal{S}$-Ramsey and $\mathcal{S}$-Baire subsets are both $\sigma$-algebras, they coincide, and they are closed under the Souslin $\mathcal{A}$-operation. Moreover, the ideals of $\mathcal{S}$-Ramsey null and $\mathcal{S}$-meager subsets are $\sigma$-ideals and also coincide.

Remark 34 . One can easily check the basic clopen subsets of $\mathcal{R}$ are $\mathcal{S}$-Baire. Hence we can conclude from the main Theorem 33 that every Borel subset of $\mathcal{R}$ is $\mathcal{S}$ Ramsey.

Definition 35. Any structure of the form $\left(\mathcal{R}, \mathcal{S}, \leq, \leq^{0}, p, q\right)$ satisfying the conclusion of Theorem 33 is called a Ramsey space.

$$
\text { 5. The Ramsey } \operatorname{space}\left(C_{f}^{*}, C_{p}^{*}, \subseteq, \subseteq, p, q\right)
$$

In this section we will apply the abstract Ramsey theory presented in the previous section in the context of subtrees of $C$.

Let $f, p$ be tree codings such that $p$ is in addition perfect. We set $\mathcal{R}=C_{f}^{*}$ and $\mathcal{S}=C_{p}^{*}$. We will define the approximations $p=\left(p_{n}\right)_{n}$ and $q=\left(q_{n}\right)_{n}$ on $C_{f}^{*}$ and $C_{p}^{*}$ respectively, and we will verify Axioms 1-6 in the resulting space $\left(C_{f}^{*}, C_{p}^{*}, \subseteq, \subseteq, p, q\right)$.

Definition 36. For every $A \in C_{f}^{*}, T \in C_{p}^{*}$ we define

(a) $p_{0}(A)=\varnothing, p_{n}(A)=\left.\bar{A}\right|_{n}=\bigcup_{k<n} \bar{A}(k), n \geq 1$.

(b) $q_{0}(T)=\varnothing, q_{n}(T)=\left.\bar{T}\right|_{n}=\bigcup_{k<n} \bar{T}(k), n \geq 1$,

where $\bar{A}$ and $\bar{T}$ are the regular envelopes of $A$ and $T$, respectively. 
We set $\mathcal{P}_{n}$ and $\mathcal{Q}_{n}$ to be the ranges of $p_{n}$ and $q_{n}$, respectively. In addition we set $\mathcal{P}=\bigcup_{n} \mathcal{P}_{n}$ and $\mathcal{Q}=\bigcup_{n} \mathcal{Q}_{n}$. Also let $\bar{f}, \bar{p}, \sigma_{f}, \sigma_{p}$ be the regular envelopes and the level parameterizations of $f$ and $p$.

Observe that $p_{n}(A)$ and $q_{n}(T)$ are not subsets of $A$ and $T$ and actually are the $n^{\text {th }}$ approximations of their regular envelopes which belong to $C_{\bar{f}}$ and $C_{\bar{p}}$, respectively. But this will cause no problem in view of Propositions 17 and 23. As we will see, the passing from $C_{f}^{*}$ to $C_{\bar{f}}$ plays a key role in the verification of Axioms 1-6.

Notation. Let $f$ be a tree coding. Recall that by $C_{f}$ we denote the set of all regular $f$-subtrees of $C$.

(a) For every $d \in \mathbb{N}$, we set $C_{f}^{d}=\left\{a \subseteq C: \exists R \in C_{f}\right.$ such that $\left.a=\left.R\right|_{d}\right\}$, where $\left.R\right|_{d}=\bigcup_{n<d} R(n)$

(b) Let $\sigma_{f}$ be the level parameterization of $f$ and let $a \in C_{f}^{d}$, where $d \in \mathbb{N}$. For each $n<d$, let $a(n)=\left\{t_{0}^{n} \prec \cdots \prec t_{k_{n}}^{n}\right\}$ be the lexicographic ordering of $a(n)$. Then we set $\sigma_{f}(a)=\left\{t_{\sigma_{f}(n)}^{n}: 0 \leq n<d\right\}$.

Proposition 37. The space $\left(C_{f}^{*}, C_{p}^{*}, \subseteq, \subseteq, p, q\right)$ satisfies Axioms 1-3.

Proof. Axioms 1 and 3 hold trivially. To see that Axiom 2 is also satisfied, let $A, B \in C_{f}^{*}$ such that $p_{d}(A)=p_{d}(B)$ for all $d$. Then $\bar{A}=\bar{B}$ and so $A=\sigma_{f}(\bar{A})=$ $\sigma_{f}(\bar{B})=B$. Similarly for $T, S \in C_{p}^{*}$.

Definition 38. For every $a \in \mathcal{P}, u, u^{\prime} \in \mathcal{Q}$ we define

(a) $a \leq_{\text {fin }}^{0} u$ iff $a \subseteq u$ and $\sigma_{f}(a) \subseteq \sigma_{p}(u)$.

(b) $u^{\prime} \leq_{\text {fin }} u$ iff $u^{\prime} \subseteq u$ and $\sigma_{p}\left(u^{\prime}\right) \subseteq \sigma_{p}(u)$.

Proposition 39. The relations $\leq_{\text {fin }}^{0}, \leq_{\text {fin }}$ are finitizations of the inclusion $\subseteq$ on $C_{f}^{*} \times C_{p}^{*}$ and on $C_{p}^{*} \times C_{p}^{*}$, respectively.

Proof. Let $A \in C_{f}^{*}, T \in C_{p}^{*}$ such that $A \subseteq T$. Then $\bar{A} \subseteq \bar{T}$, and since both are regular subtrees of $C$, we have that $\bar{A}$ is a regular subtree of $\bar{T}$. So there exists a strictly increasing sequence $\left(m_{k}\right)_{k}$ such that for every $k, \bar{A}(k) \subseteq \bar{T}\left(m_{k}\right)$. Then for every $n, p_{n}(A) \subseteq q_{m_{n}}(T)$ and if $a=p_{n}(A)$ and $u=q_{m_{n}}(T)$, then $\sigma_{f}(a)=a \cap A \subseteq u \cap T=\sigma_{p}(u)$. Therefore if $A \subseteq T$, then for every $n$ there exists an $m$ such that $p_{n}(A) \leq_{f i n}^{0} q_{m}(T)$. Conversely, if the latter holds, then for every $n$ $\sigma_{f}\left(\left.\bar{A}\right|_{n}\right) \subseteq T$ and so $A \subseteq T$. Similarly we prove that $\leq_{\text {fin }}$ is a finitization of $\subseteq$ on $C_{p}^{*} \times C_{p}^{*}$.

The proof of the following proposition is simple and so we omit it.

Proposition 40. The space $\left(C_{f}^{*}, C_{p}^{*}, \subseteq, \subseteq, p, q\right)$ satisfies Axiom 4 .

Notation. Let $W$ be a perfect subtree of $C$. For all $d, a \in C \frac{d}{f}, u \in C_{\bar{p}}^{d}$ we define

$$
\begin{aligned}
& {[a, W]=\left\{A \in C_{f}^{*}: a=p_{d}(A) \text { and } A \subseteq W\right\},} \\
& {[u, W]=\left\{T \in C_{p}^{*}: u=q_{d}(T) \text { and } T \subseteq W\right\} .}
\end{aligned}
$$

Lemma 41. Let $W$ be a perfect subtree of $C, d \in \mathbb{N}, a \in C \frac{d}{f}$ and $u \in C \frac{d}{p}$, where $\bar{f}$ and $\bar{p}$ are the regular envelopes of $f$ and $p$. Then:

(a) $[a, W] \neq \varnothing$ iff $a \subseteq \bar{W}$ and $\sigma_{f}(a) \subseteq W$.

(b) $[u, W] \neq \varnothing$ iff $u \subseteq \bar{W}$ and $\sigma_{p}(u) \subseteq W$. 
Proof. We will prove only part (a) (the proof of part (b) is similar). If $[a, W] \neq \varnothing$, then for every $A \in[a, W], p_{d}(A)=a$ and $A \subseteq W$; that is, $a \subseteq \bar{A} \subseteq \bar{W}$ and $\sigma_{f}(a) \subseteq \sigma_{f}(\bar{A})=A \subseteq W$. Conversely, suppose that $a \subseteq \bar{W}$ and $\sigma_{f}(a) \subseteq W$. By induction, we construct a sequence $a=a_{0} \subseteq a_{1} \subseteq \ldots$ such that for every $n$, $a_{n} \in C_{\bar{f}}^{d+n}, a_{n} \subseteq \bar{W}$ and $\sigma_{f}\left(a_{n}\right) \subseteq W$. The construction can be easily done. We set $R=\bigcup_{n} a_{n}$. Then $R \in C_{\bar{f}}$, and if $A=\sigma_{f}(R)$, then $A \in[a, W]$.

By the above lemma, we get the following.

Corollary 42. Let $W$ be a perfect subtree of $C, d \in \mathbb{N}$ and $a \in C \frac{d}{f}$ such that $[a, W] \neq \varnothing$. Then

$$
p_{d+1}^{\prime \prime}[a, W]=\left\{a^{\prime} \in C_{\bar{f}}^{d+1}: a \subseteq a^{\prime} \subseteq \bar{W} \text { and } \sigma_{f}\left(a^{\prime}\right) \subseteq W\right\} .
$$

We also have the following easily verified remark.

Remark 1. Suppose that $d \geq 1, a \in \mathcal{P}_{d}, T \in C_{p}^{*}$ and $[a, T] \neq \varnothing$. Then $n=$ $\operatorname{depth}_{T}(a) \geq d$ and $a(d-1) \subseteq u(n-1)$, where $u=q_{n}(T)$.

Proposition 43. The space $\left(C_{f}^{*}, C_{p}^{*}, \subseteq, \subseteq, p, q\right)$ satisfies Axiom 5 .

Proof. Let us first show that the space $\left(C_{f}^{*}, C_{p}^{*}, \subseteq, \subseteq, p, q\right)$ satisfies Axiom 5(i). So let $a \in \mathcal{P}, T \in C_{p}^{*}$ such that $[a, T] \neq \varnothing$ and $S \in[n, T]$, where $n=\operatorname{depth}_{T}(a)$. Let $u=q_{n}(T)=q_{n}(S)$. Then $a \leq_{f i n}^{0} u$ and so $a \subseteq u \subseteq \bar{S}, \sigma_{f}(a) \subseteq \sigma_{p}(u) \subseteq S$. By Lemma 41(a), we have that $[a, S] \neq \varnothing$.

We will now verify Axiom 5(ii). Let $a \in \mathcal{P}$ and $S, T \in C_{p}^{*}$ such that $S \subseteq T$, $[a, T] \neq \varnothing$ and $[a, S] \neq \varnothing$. We have to show that there exists a $T^{\prime} \in[n, T]$, $n=\operatorname{depth}_{T}(a)$, such that $\left[a, T^{\prime}\right] \subseteq[a, S]$. Since $[a, T] \neq \varnothing$, we have that $n \geq 0$. If $n=0$, then we can set $T^{\prime}=S$. Otherwise, let $u=q_{n}(T)$. By Remark 1 , we have that $a(d-1) \subseteq u(n-1)$ and since $[a, S] \neq \varnothing, a(d-1) \subseteq \bar{S}$. For each $\bar{s} \in a(d-1)$ and $\bar{t} \in u(n-1) \backslash a(d-1)$, we set

$$
S_{\bar{s}}=\{s \in S: s \geq \bar{s}\} \text { and } T_{\bar{t}}=\{t \in T: t \geq \bar{t}\} .
$$

Let $S^{\prime}=\bigcup\left\{S_{\bar{s}}: \bar{s} \in a(d-1)\right\}$ and $T^{\prime}=\bigcup\left\{T_{\bar{t}}: \bar{t} \in u(n-1) \backslash a(d-1)\right\}$. We set

$$
W=\sigma_{p}(u) \cup S^{\prime} \cup T^{\prime} .
$$

Then it is easily verified that $W$ is a perfect subtree of $T, u \subseteq \bar{W}$ and so by Lemma 41(b), there exists a $T^{\prime} \in[u, W] \subseteq[n, T]$. Also note that $\left[a, T^{\prime}\right] \subseteq[a, W] \subseteq$ $[a, S]$.

Theorem 44. The space $\left(C_{f}^{*}, C_{p}^{*}, \subseteq, \subseteq, p, q\right)$ is a Ramsey space.

Proof. By the above propositions it remains to prove that Axiom 6 also holds. Let $d \in \mathbb{N}, \mathcal{Y} \subseteq \mathcal{P}_{d+1}, a \in \mathcal{P}_{d}$ and $T \in C_{p}^{*}$ such that $[a, T] \neq \emptyset$. We have to prove that there exists $S \in\left[n_{0}, T\right]$ where $n_{0}=\operatorname{depth}_{T}(a)$, such that either $p_{d+1}^{\prime \prime}[a, S] \subseteq \mathcal{Y}$ or $p_{d+1}^{\prime \prime}[a, S] \subseteq \mathcal{Y}^{c}$.

We will treat the case $n_{0} \geq 1$ or equivalently $a \neq \emptyset$, since the case $a=\emptyset$ is an easy application of HLLP Theorem for a single tree. So let $\bar{s}_{0}, \ldots, \bar{s}_{m}$ be the lexicographic increasing enumeration of $a(d-1)$ and let $k=\left|\bar{s}_{\sigma_{f}(d-1)}\right|_{T}$, that is, $k$ is the order in $T$ of the unique node of the last level of $a$ which belongs to $T$. For $i=0 \ldots, m$, we set

$$
T_{i}=\left\{t \in T: t>\bar{s}_{i} \text { and }|t|_{T} \geq k+1\right\} \cup\left\{\bar{s}_{i}\right\} .
$$


Then each $T_{i}$ is a perfect subtree of $T$ with root $\bar{s}_{i}$ such that $T_{i}(n) \subseteq T(k+n)$ for $n \geq 1$.

Let $\bar{f}$ be the regular envelope of $f$ and $i_{0}=\sigma_{f}(d)$. Then by Lemma 21, $0 \leq i_{0} \leq$ $m$. For each $t \in T_{i_{0}}(n), n \geq 1$, we define

$$
\phi_{t}: T(k+n) \rightarrow \hat{T}(\ell), \quad \ell=|t|,
$$

by $\phi_{t}(t)=t$ and for all $t^{\prime} \in T(k+n)$ with $t^{\prime} \neq t$,

(i) If $t^{\prime} \prec t$, then we set $\phi_{t}\left(t^{\prime}\right)=t^{\prime \prime} \uparrow \ell$, for all $t^{\prime \prime} \in \operatorname{ImmSucc}\left(t^{\prime}, T\right)$.

(ii) If $t \prec t^{\prime}$, then we set $\phi_{t}\left(t^{\prime}\right)=t^{\prime} \uparrow \ell$.

Since $T$ is rapidly increasing, by Lemma 18 , the above map is well defined.

Let $\bar{f}(d-1)=\left(\varepsilon_{0} \ldots \varepsilon_{m}\right)$. By Lemma 21, we distinguish the following two possible cases.

Case 1. For all $i=0, \ldots, m, \varepsilon_{i}=1$.

Then we define

$$
\Phi_{1}: \otimes_{n \geq 1}\left(T_{0}, \ldots, T_{i_{0}}, \ldots, T_{m}\right) \rightarrow p_{d+1}^{\prime \prime}[a, T]
$$

by

$$
\Phi_{1}\left(\left(t_{0}, \ldots, t_{i_{0}}, \ldots, t_{m}\right)\right)=a \cup \phi_{t_{0}}^{\prime \prime}\left\{t_{0}, \ldots, t_{i_{0}}, \ldots, t_{m}\right\} .
$$

By Corollary 42, $\Phi_{1}$ is well defined. We set $K=\Phi_{1}^{-1}(\mathcal{Y})$ and $K^{c}=\otimes\left(T_{0}, \ldots, T_{m}\right) \backslash$ $\Phi_{1}^{-1}(\mathcal{Y})$. By Theorem 10 for $\varepsilon=1$, there exists a regular perfect vector subtree $\left(S_{0}, \ldots, S_{m}\right)$ of $\left(T_{0}, \ldots, T_{m}\right)$ such that

$$
\text { either } \otimes\left(S_{0}, \ldots, S_{m}\right) \subseteq K \quad \text { or } \quad \otimes\left(S_{0}, \ldots, S_{m}\right) \subseteq K^{c} .
$$

We set

$$
u=q_{n_{0}}(T), \quad S^{\prime}=\bigcup_{i=0}^{m} S_{i} \backslash\left\{\bar{s}_{i}\right\}, \quad T^{\prime}=\bigcup\left\{T_{\bar{t}}: \bar{t} \in u\left(n_{0}-1\right) \backslash a(d-1)\right\}
$$

and we define

$$
W=\sigma_{p}(u) \cup S^{\prime} \cup T^{\prime}
$$

It is clear that $W$ is a perfect subtree of $C$ such that $u \subseteq \bar{W}$. From the fact that $\left(S_{0}, \ldots, S_{m}\right)$ is a regular vector subtree of $\left(T_{0}, \ldots, T_{m}\right)$, we have that

$$
\Phi_{1}^{\prime \prime} \otimes\left(S_{0}, \ldots, S_{m}\right)=p_{d+1}^{\prime \prime}[a, W] .
$$

Therefore, either $p_{d+1}^{\prime \prime}[a, W] \subseteq \mathcal{Y}$ if $\otimes\left(S_{0}, \ldots, S_{m}\right) \subseteq K$, or $p_{d+1}^{\prime \prime}[a, W] \subseteq \mathcal{Y}^{c}$, otherwise.

By Lemma 41(b), there exists an $S \in[u, W]$. Then $S \in[n, T],[a, S] \subseteq[a, W]$ and so either $p_{d+1}^{\prime \prime}[a, S] \subseteq \mathcal{Y}$ or $p_{d+1}^{\prime \prime}[a, S] \subseteq \mathcal{Y}^{c}$.

Case 2. For all $i=0, \ldots, m$ with $i \neq i_{0}, \varepsilon_{i}=1$ and $\varepsilon_{i_{0}}=2$.

Then we define

$$
\Phi_{2}: \otimes\left(T_{0}, \ldots, T_{i_{0}}^{(2)}, \ldots, T_{m}\right) \rightarrow p_{d+1}^{\prime \prime}[a, T]
$$

by

$$
\Phi_{2}\left(\left(t_{0}, \ldots,\left\{t_{i_{0}}^{0} \prec t_{i_{0}}^{1}\right\}, \ldots, t_{m}\right)\right)=a \cup \phi_{t_{i_{0}}^{0}}^{\prime \prime}\left\{t_{0}, \ldots, t_{i_{0}}^{0}, t_{i_{0}}^{1}, \ldots, t_{m}\right\} .
$$

As in Case $1, \Phi_{2}$ is well defined. We set $K=\Phi_{2}^{-1}(\mathcal{Y})$ and by Theorem 10 for $\varepsilon=2$, there exists a regular perfect vector subtree $\left(S_{0}, \ldots, S_{m}\right)$ of $\left(T_{0}, \ldots, T_{m}\right)$ such that either $\otimes\left(S_{0}, \ldots, S_{i_{0}}^{(2)}, \ldots, S_{m}\right) \subseteq K$ or $\otimes\left(S_{0}, \ldots, S_{i_{0}}^{(2)}, \ldots, S_{m}\right) \subseteq K^{c}$. Then we proceed in a similar way as in Case 1 . 
By Remark [34 we have that the basic clopen subsets

$$
[a]=\left\{A \in C_{f}^{*}: \exists n \text { such that } p_{n}(A)=a\right\}
$$

of $C_{f}^{*}$ are $C_{p}^{*}$-Ramsey. It can be easily checked that the above subsets form a basis for the induced topology of $C_{f}^{*}$ as a subspace of $2^{C}$. Also since $C_{f}^{*}$ is closed in $\mathcal{P}^{\mathbb{N}}, C_{f}^{*}$ is a Polish space and therefore every analytic subset is the image of the Souslin operation on a sequence of closed subsets of $C_{f}^{*}$ (see for example $[\mathbf{K}]$ ). Hence by Theorem 44 every analytic subset $K$ of $C_{f}^{*}$ is $C_{p}^{*}$-Ramsey. Since by Proposition 15 every perfect subtree of $C$ contains a member of $C_{p}^{*}$, we get the following consequence of Theorem 44, which establishes Theorem A of the Introduction.

Theorem 45. Let $f$ be a tree coding and let $C_{f}^{*}=K_{0} \cup K_{1}$, where $K_{0}$ is analytic and $K_{1}$ is co-analytic. Then for every perfect subtree $T$ of $C$ there exists a perfect subtree $T^{\prime}$ of $T$ such that either $\left[T^{\prime}\right]_{f}^{*} \subseteq K_{0}$ or $\left[T^{\prime}\right]_{f}^{*} \subseteq K_{1}$, where $\left[T^{\prime}\right]_{f}^{*}=\left\{A \in C_{f}^{*}\right.$ : $\left.A \subseteq T^{\prime}\right\}$.

5.1. Notes. Similar results to Theorem 44 hold for the case of regular trees. Let us state them briefly. Let $f$ be a skew tree coding, let $p$ be a perfect tree coding and let $C_{f}, C_{p}$ be the sets of regular $f$-subtrees and $p$-subtrees of $C$, respectively. Let for each $d \in \mathbb{N}, A \in C_{f}, p_{d}(A)=\left.A\right|_{d}$ and for each $n \in \mathbb{N}, T \in C_{p}, q_{n}(T)=\left.T\right|_{n}$. Define the finitizations to be the inclusion ordering. Then we have the following.

Theorem 46. For every skew tree coding $f$ and perfect coding $p$, the space

$$
\left(C_{f}, C_{p}, \subseteq, \subseteq, p, q\right)
$$

is a Ramsey space.

The verification of Axioms 1-5 is easily established and that of Axiom 6 is immediately derived from Theorem 10. Setting $f(n)=(1)$ for all $n$, that is, $f$ is the coding of a chain in $C$ and $C_{f}$ is the set of all chains of $C$, we obtain the parameterized Ellentuck theorem due to Todorčević, A. W. Miller [Mi] and J. Pawlikowski $[\mathrm{P}$. Also by taking envelopes Theorem [46] can derive the Louveau-Shelah-Veličković Theorem.

As a matter of fact, one can also prove an extension of Theorem 46 concerning finitely branching regular subtrees of a perfect tree. Let us point out that at first glance a partition theorem for non-skew regular subtrees seems to be impossible. For instance (as in an example in subsection 2.2), let $f$ be the tree coding given by $f(0)=(2), f(1)=(2,2)$ and $f(n)=(1,1,1,1)$ for all $n \geq 2$ and consider the subset $\mathcal{U}$ of $C_{f}$ defined by

$$
A \in \mathcal{U} \text { iff } A(2)=\left\{t_{1} \prec t_{2} \prec t_{3} \prec t_{4}\right\} \text { and }\left|t_{1} \wedge t_{2}\right|<\left|t_{3} \wedge t_{4}\right| .
$$

Then $\mathcal{U}$ is an open subset of $C_{f}$, but it is easily seen that for every perfect subtree $T$ of $C,\left\{A \in C_{f}: A \subseteq T\right\}$ has non-empty intersection with both $\mathcal{U}$ and $C_{f} \backslash \mathcal{U}$.

A careful examination of the above example shows that the family $\mathcal{U}$ contains trees whose envelopes in $C$ are of different types. Note that in the case of regular skew $f$-subtrees, these kinds of pathologies do not occur, since as it can be easily seen that the type of the envelope of a skew tree is completely determined by its coding.

To overcome these difficulties, we restrict ourselves to families of regular finitely branching subtrees of a perfect tree $T$, which not only are mutually strongly isomorphic but in addition the envelope of them in $C$ is a skew tree of a fixed type. 
To be more precise let us call a type $\tau$ compatible to a tree coding $f$, if there exists a regular $f$-subtree $A$ of $C$ such that $\hat{A}$ is of type $\tau$. Let us denote by $C_{(f, \tau)}$ the set of all regular $f$-subtrees of $C$, such that their envelope is of type $\tau$. Then we have the following extension of Theorem 46 .

Theorem 47. Let $f$ be a tree coding, let $\tau$ be a compatible to $f$ type and let $p$ be a perfect coding. Then the space $\left(C_{(f, \tau)}, C_{p}, \subseteq, \subseteq, p, q\right)$ is a Ramsey space.

The verification of Axioms 1-5 for the above theorem is again easily obtained. We sketch the proof that Axiom 6 is also valid. It can be shown that there exists a skew tree coding $g$ and an infinite subset $L$ of $\mathbb{N}$, depending on $f$ and $\tau$, such that for every $A^{\prime} \in C_{(f, \tau)}$ there exists an $A \in C_{g}$ such that $\left.\hat{A}\right|_{L}=A^{\prime}$. Under this remark and using Theorem 46, one can show that Axiom 6 also holds.

\section{ACKNOWLEDGEMENTS}

I am indebted to Professor S.A. Argyros for his interest, suggestions and valuable help during the preparation of the paper. Also, I would like to thank Drs. V. Felouzis and P. Dodos for their continuous support and encouragement.

\section{REFERENCES}

[AFK] S.A. Argyros, V. Felouzis and V. Kanellopoulos, A proof of Halpern-Läuchli Partition Theorem, Europ. J. Combinatorics, 23(2002), 1-10. MR 1878768 (2002k:05058)

[B] A. Blass, A partition theorem for perfect sets, Proc. AMS, 82(1981), 271-277. MR0609665 (83k:03063)

[C] T.J. Carlson, Some unifying principles in Ramsey theory, Discrete Math., 68(1988), 117169. MR0926120 (89b:04006)

[CS] T.J. Carlson and S.G. Simson, A dual form of Ramsey's theorem, Adv. in Math., 53(1984), 265-290. MF 0753869 (85h:04002)

[E] E. Ellentuck, A new proof that analytic sets are Ramsey, J. Symb. Logic, 39(1974), 163165. MR0349393 (50:1887)

[G] F. Galvin, Partition theorems for the real line, Notices AMS, 15(1968), 660.

[GP] F. Galvin and K. Prikry, Borel sets and Ramsey's theorem, J. Symb. Logic, 38(1973), 193-198. MF0337630(49:2399)

[H] J.D. Halpern, Nonstandard combinatorics, Proc. London Math. Soc., 30(1975), 40-54. MR0389605 (52:10436)

[HL] J.D. Halpern and H. Läuchli, A partition theorem, Trans. AMS, 124(1966), 360-367. MR0200172(34:71)

[HP] J.D. Halpern and D. Pincus, Partitions of products, Trans. AMS, 267(1981), 549-568. MR0626489 (83b:03058)

[K] A.S. Kechris, Classical Descriptive Set Theory, Springer, 1995. MR1321597 (96e:03057)

[L] R. Laver, Products of infinitely many perfect trees, J. London Math. Soc., 29(1984), 385396. MR0754925 (85j:03078)

[LSV] A. Louveau, S. Shelah and B. Veličković, Borel partitions of infinite trees of a perfect tree, Annals of Pure and Appl. Logic, 63(1993), 271-281. MR1237234 (94g:04003)

[Mi] A.W. Miller, Infinite combinatorics and definability, Annals of Pure and Appl. Logic, 41(1989), 179-203. MR0983001 (90b:03070)

[M1] K. Milliken, A partition theorem for the infinite subtrees of a tree, Trans. AMS, 263(1981), 137-148. MR0590416 (82g:04003)

[M2] K. Milliken, A Ramsey theorem for trees, J. Comb. Theory A, 26(1979), 215-237. MR0535155 (80j:05090)

[P] J. Pawlikowski, Parametrized Ellentuck theorem, Topology and its Appl., 37(1990), 65-73. MR.1075374 (91j:04002)

[St] J. Stern, A Ramsey theorem for trees, with an application to Banach spaces, Israel J. Math., 29(1978), 179-188. MR0476554(57:16114) 
[T1] S. Todorčević, Compact subsets of the first Baire class, Journal AMS, 12(1999), 1179-1212. MR.1685782 (2000d:54028)

[T2] S. Todorčević, Lectures Notes in Infinite Dimensional Ramsey Theory, (manuscript) University of Toronto, 1998 .

Department of Mathematics, National Technical University of Athens, Athens 15780 , GREECE

E-mail address: bkanel@math.ntua.gr 